\title{
THE STRAIN OF A GRAVITATING, COMPRESSIBLE ELASTIC
}

\section{SPHERE*}

LY

\section{M. HOSKINS}

In the solution of the problem of the strain of a homogeneous elastic sphere, as given by LAME and by KeLvin, the bodily forces are supposed expressible as known functions of the coördinates of position. When self-gravitation is among the forces considered, however, the bodily forces depend in part upon the state of strain, and it is only in the case of assumed incompressibility that the usual method of solution is applicable.

The present paper has for its main object the solution of the problem of the strain of an isotropic elastic sphere, initially homogeneous, due to disturbing forces of a certain type, taking into account the changes in the gravitational forces which result from the strain.

The contents of the paper fall under the following four heads:

I. Strain of a gravitating, compressible elastic sphere under the action of tidal or centrifugal forces, with numerical computations for the case in which the surface is free from stress.

II. Strain of a gravitating, compressible elastic sphere covered by a shallow ocean, under the action of tidal or centrifugal forces.

III. Effect of compressibility upon estimates of the rigidity of the earth.

IV. Strain of a gravitating, compressible elastic sphere under the action of small disturbing forces derivable from a potential which is any spherical harmonic of degree not less than 2 .

\section{Strain of a COMPREssible, gravitating Elastic SPHERE UNDER The ACTION OF TIDAL OR CENTRIFUGAL FORCES. \\ §1. Physical analysis of the problem.}

The object of the analysis is to determine the strain produced in an elastic sphere by the action of disturbing forces of the type of tidal or centrifugal forces -i. e., forces derivable from a potential proportional to $P_{2} r^{2}$, in which $P_{2}$ is the zonal surface harmonic ${ }_{2}^{3} \cos ^{2} \theta-{ }_{2}^{1}$. The disturbing forces are assumed small in comparison with the actual forces of gravity, but the changes in the gravita-

\footnotetext{
* Presented to the American Mathematical Society (San Francisco), September 25, 1909.
} 
tional forces due to the strain are assumed to be of the same order as the disturbing forces. This accords with the facts in the case of the actual tidal and centrifugal forces acting upon the earth.

Before the action of the disturbing forces the body is assumed to be in equilibrium under the action of the mutual gravitation of its parts without surface stress. The stress-condition at any interior point need not be specified more definitely ; it may be hydrostatic equilibrium, or any departure from that condition which is consistent with the forces of self-attraction. The stresses and strains considered in the following analysis are to be regarded as superposed upon the supposed initial condition, it being assumed that small departures from this initial condition follow the laws of elasticity as ordinarily given for a homogeneous isotropic solid.*

When the external disturbing forces come into action, the body assumes a new configuration of equilibrium. The gravitational force which now acts upon a given element of volume is different from that which originally acted upon the same element, for two reasons, - because the density of the attracted element is changed, and because the density-distribution of the attracting mass is changed. In computing the new value of the gravitational force the new configuration of the body may be treated as made up of two parts, - the original configuration, and a certain mass-distribution superposed upon it. The total attraction upon a volume-element may then be expressed as the resultant of the following parts : (1) The attraction of the original mass-configuration upon the original mass of the element ; $(2)$ the attraction of the original mass-configuration upon the increment of mass of the element ; (3) the attraction of the increment of mass-distribution upon the original mass of the element; and (4) the attraction of the increment of mass-distribution upon the increment of mass of the element. Of these four forces (1) is balanced by the initial stresses and may be omitted from the equations of equilibrium of the element; (2) and (3) are of the same order of magnitude as the disturbing forces; while (4) is negligible in comparison with (2) and (3). Hence (2) and (3) are the only bodily forces to be considered in addition to the external disturbance.

\footnotetext{
* It has been held by certain writers that the existence of initial stresses of great magnitude vitiates the application of the theory of elasticity to a gravitating body comparable in size and mass with the earth. As to applications which are sometimes made of the theory, the objection appears to be undoubtedly valid. For example, it would be absurd to compute the actual stresses within the earth as if they were due to gravitation acting upon a body initially free from stress; even if such an assumption were in barmony with any reasonable supposition as to the history of the earth, the strains thus computed would far surpass the limits of elasticity. This objection does not, however, appear to hold against such an application of the laws of elasticity as is made in the present paper. To assume that a body under great stress, whether this is everywhere purely a normal pressure or whether involving also tangential stresses, is elastic for small departures from the initial configuration, is not inconsistent with what is known regarding bodies under ordinary experimental conditions. It can hardly be doubted that the great body of the earth is elastic as regards slight changes of configuration.
} 
The original density being called $\rho$, the increment of density $\delta \rho$ is equal to $-\rho \Delta$, if $\Delta$ is the cubical expansion. Hence the force (2) is to be expressed as the attraction of the undisturbed mass upon a density $-\rho \Delta$, while (3) is the attraction of a mass-distribution of density $-\rho \Delta$ upon a density $\rho$, together with the attraction due to the surface inequality produced by the strain.

Besides the bodily forces it is necessary to consider the stress-condition at the surface. Upon the actual bounding surface it is assumed that the stress remains zero; but if (as is convenient) the equations of interior equilibrium be assumed to apply throughout the original spherical volume, the surface of this volume must be regarded as sustaining a stress whose value must be properly expressed. It is shown below that, to the first order of small quantities, this stress is radial and equal to the weight of the surface inequality produced by the strain.

\section{\$2. Formation of equations of equilibrium for an element of volume.}

(a) General form of equations in terms of polar coördinates. Let the axis of symmetry of the disturbing forces be taken as initial line of a system of polar coördinates, the displacements in the three primary directions at a point $(r, \theta, \phi)$ being called

$$
u_{r}, \quad u_{\theta}, \quad u_{\phi},
$$

while $\Delta$ is the cubical expansion. In the case now under consideration

$$
u_{\phi}=0 \text {. }
$$

Let the total bodily force per unit volume have components

(the $\phi$-component being 0 ).

$$
\rho F_{r}, \quad \rho F_{\theta},
$$

In accordance with a common notation, let $\mu$ denote the modulus of distortion and $\lambda+\frac{2}{3} \mu$ the bulk-modulus of the material. The equations of equilibrium for an elementary volume then assume the following forms: * in which

$$
\begin{gathered}
(\lambda+2 \mu) \frac{\partial \Delta}{\partial r}-\frac{\mu}{r^{2} \sin \theta} \partial\left[\begin{array}{c}
\partial \theta \\
\partial \theta
\end{array}\left[\left(\frac{\partial\left(r u_{\theta}\right)}{\partial r}-\frac{\partial u_{r}}{\partial \theta}\right) \sin \theta\right]+\rho F_{r}=0\right. \\
(\lambda+2 \mu) \frac{1}{r} \frac{\partial \Delta}{\partial \theta}+\frac{\mu}{r \sin \theta} \frac{\partial}{\partial r}\left[\left(\frac{\partial\left(r u_{\theta}\right)}{\partial r}-\frac{\partial u_{r}}{\partial \theta}\right) \sin \theta\right]+\rho F_{\theta}=0 ;
\end{gathered}
$$

$$
\Delta=\frac{1}{r^{2} \sin \theta}\left[\frac{\partial}{\partial r}\left(r^{2} u_{r} \sin \theta\right)+\frac{\partial}{\partial \theta}\left(r u_{\theta} \sin \theta\right)\right] .
$$

(b) Assumptions. It is found that by making certain assumptions the equations are reduced to a form admitting of solution. It will be well to state these

* See A. E. H. Love's A Treatise on the Mathenatical Theory of Elasticity, 2d edition (1906), pp. 138, 56. The terms representing bodily forces are omitted from the equations as given on p. 138. 
assumptions explicitly, at the same time remarking that their justification is the fact that they lead to a solution satisfying all the conditions of the problem.

(1) The potential of the disturbing forces being proportional to $r^{2} P_{2}$, in which $P_{2}=\frac{3}{2} \cos ^{2} \theta-\frac{1}{2}$, it is assumed that the radial displacement and the cubical expansion depend upon $\theta$ in the same way as this potential does ; i. e., each is assumed to be the product of $P_{2}$ by a function of $r$.

(2) It is assumed that the mean radius of an originally spherical surface is unchanged by the strain.

(c) Linear displacements replaced by ellipticity and maximum angular displacement. It is found convenient to replace $u_{r}$ and $u_{\theta}$ in the foregoing equations by two quantities $e, \alpha$, defined as follows:

The former represents the ellipticity of the surface originally spherical and of radius $r$; that is,

$$
u_{r}=-\frac{2}{3} e r P_{2}
$$

(it being assumed that a positive value of $e$ corresponds to oblateness).

The quantity $\alpha$ represents the angular displacement of a radius vector for which $\theta=45^{\circ}$. It is then found that, for any point $(r, \theta)$,

$$
\frac{u_{\theta}}{r}=\alpha \sin 2 \theta \text {. }
$$

This follows from the assumption that $\Delta$ involves $\theta$ only in a factor $P_{2}$.

To make the last statement clear, notice that the introduction of $e$ in place of $u_{r}$ reduces (3) to the form

$$
\Delta=-\left(2 e+\frac{2}{3} r \frac{d e}{d r}\right) P_{2}+\frac{1}{r \sin \theta} \frac{\partial\left(u_{\theta} \sin \theta\right)}{\partial \theta} .
$$

In order that the last term may be equivalent to $P_{2}$ multiplied into a function of $r$, it is easy to show that we must have

$$
u_{\theta}=\alpha \sin 2 \theta+\frac{\beta}{\sin \theta},
$$

in which $\alpha$ and $\beta$ are independent of $\theta$. Infinite values of $u_{\theta}$ being excluded, $\beta$ must be $0 . *$

If, now, $u_{r}$ and $u_{\theta}$ be replaced by $e$ and $\alpha$. by means of (4) and (5), equations (1) and (2) take the following forms:

* It is otherwise evident that, for a given value of $r, u_{\theta}$ will have equal values for complementary values of $\theta$. This is seen by considering the effect of superposing strains of the same type about different axes. Thus the $\%$-displacements in a given meridian plane (say that of $x z$ ) will be annulled by superposing upon the given strain either $(a)$ an exactly similar strain symmetrical about the $x$-axis, or $(b)$ a strain symmetrical about the $z$-axis which is just the reverse of the given strain. 
(6) $\left[4(\lambda+\mu) \frac{d \alpha}{d r}-8 \mu \frac{\alpha}{r}-\frac{2}{3}(\lambda+2 \mu) r \frac{d^{2} e}{d r^{2}}-\frac{8}{3}(\lambda+2 \mu) \frac{d e}{d r}+4 \mu \frac{e}{r}\right] P_{2}+\rho F_{r}=0$, (7) $\left[\mu r \frac{d^{2} \alpha}{d r^{2}}+4 \mu \frac{d \alpha}{d r}-2(3 \lambda+5 \mu) \frac{\alpha}{r}+(\lambda+\mu) \frac{d e}{d r}+(3 \lambda+5 \mu) \frac{e}{r}\right] \sin 2 \theta+\rho F_{\theta}=0$, while the value of $\Delta$ becomes

$$
\Delta=\left(4 x-2 e-\frac{2}{3} r \frac{d e}{d r}\right) P_{2} .
$$

(d) Expression of bodily forces. The bodily forces acting upon an elementary volume are (1) the disturbing force (here supposed to be the centrifugal force due to a uniform rotation about a fixed axis), (2) the gravitational force due to the change of density of the attracted element, (3) the attraction due to the change of configuration of the attracting mass. We proceed to get expressions for these in terms of $r, \theta, e$ and $\alpha$.

(1) The disturbing force.

Representing the angular velocity of the rotation by $\omega$, the centrifugal forces have a potential

$$
W=-\frac{1}{3} \omega^{2} r^{2} P_{2}
$$

(neglecting a term independent of $\theta$ ). If $a$ is the radius of the sphere, $g$ the value of gravity at the surface, and $c$ the ratio of equatorial centrifugal force to gravity, i. e.,

we may write

$$
c=\frac{\omega^{2} a}{g}
$$

$$
W=-\frac{c g}{3 a} r^{2} P_{2} \text {. }
$$

The parts of $F_{r}$ and $F_{\theta}$ corresponding to this potential are

$$
\frac{\partial W}{\partial r}=-\frac{2 c g}{3 a} r P_{2}
$$

$$
\frac{1}{r} \frac{\partial W}{\partial \theta}=\frac{c g}{2 a} r \sin 2 \theta
$$

(2) Force due to change of density of attracted element.

The attraction per unit mass at any point, due to the original homogeneous sphere, is a radial force equal to

$$
-g_{\frac{r}{a}}^{r}
$$

The increment of density of the attracted element being $-\rho \Delta$, the force per unit volume is

$$
\left(-g \frac{r}{a}\right)(-\rho \Delta)=g_{a}^{\rho r \Delta} \underset{a}{ } ;
$$


hence this force is accounted for by adding to the value of $\rho F_{r}$ a term

$$
\frac{\rho g r \Delta}{a}=\rho g \frac{r}{a}\left(4 \alpha-2 e-\frac{2}{3} r \frac{d e}{d r}\right) P_{2} .
$$

(3) Force due to change of configuration of the attracting mass.

We have now to express the value of the potential at any point due to a mass-distribution of density $-\rho \Delta$ throughout the original spherical volume, together with that due to the surface layer superposed upon this sphere by reason of the strain.

Consider a shell of radius $r^{\prime}$, thickness $d r^{\prime}$, and density

$$
-\rho \Delta^{\prime}=-\rho\left(4 \alpha^{\prime}-2 e^{\prime}-\frac{2}{3} r^{\prime} \frac{d e^{\prime}}{d r^{\prime}}\right) P_{2} .
$$

The potential at a point $(r, \theta)$ due to this shell is

$$
\begin{array}{ll}
-\frac{4}{5} \pi \gamma \rho\left(4 \alpha^{\prime}-2 e^{\prime}-\frac{2}{3} r^{\prime} \frac{d e^{\prime}}{d r^{\prime}}\right) \frac{r^{2}}{r^{\prime}} d r^{\prime} \cdot P_{2} & \left(\text { if } r<r^{\prime}\right), \\
-\frac{4}{5} \pi \gamma \rho\left(4 \alpha^{\prime}-2 e^{\prime}-\frac{2}{3} r^{\prime} \frac{d e^{\prime}}{d r^{\prime}}\right) \frac{r^{4}}{r^{3}} d r^{\prime} \cdot P_{2} & \text { (if } \left.r>r^{\prime}\right) .
\end{array}
$$

Hence the potential at any point $(r, \theta)$ within the spherical volume of radius $a$, due to the density-distribution $-\rho \Delta$, is

$$
\begin{aligned}
& -\frac{4 \pi \rho P_{2}}{5}\left[\frac{1}{r^{3}} \int_{0}^{r}\left(4 \alpha^{\prime}-2 e^{\prime}-\frac{2}{3} r^{\prime} \frac{d e^{\prime}}{d r^{\prime}}\right) r^{\prime 4} d r^{\prime}\right. \\
& \left.+r^{2} \int_{r}^{a}\left(4 \alpha^{\prime}-2 e^{\prime}-\frac{2}{3} r^{\prime} \frac{d e^{\prime}}{d r^{\prime}}\right) \frac{d r^{\prime}}{r^{\prime}}\right] .
\end{aligned}
$$

To this must be added the potential due to the surface inequality. If $e_{1}$ is the surface value of $e$, the thickness of the layer is $-\frac{2}{3} e_{1} a P_{2}$, and the potential due to it at a point $(r, \theta)$ within the body is

$$
-\frac{8}{15} \pi \gamma \rho e_{1} r^{2} P_{2} \text {. }
$$

The total potential due to the change of mass-distribution may therefore be written in the following form, in which $g$ replaces $\frac{4}{3} \pi \gamma \rho a$, the surface value of gravity :

$$
V=-\frac{3 g P_{2}}{5 a}\left[\frac{1}{r^{3}} \int_{0}^{r}\left(4 \alpha^{\prime}-2 e^{\prime}-\frac{2}{3} r^{\prime} \frac{d e^{\prime}}{d r^{\prime}}\right) r^{\prime 4} d r^{\prime}\right.
$$

By partial integration we find

$$
\left.+r^{2} \int_{r}^{a}\left(4 \alpha^{\prime}-2 e^{\prime}-\frac{2}{3} r^{\prime} \frac{d e^{\prime}}{d r^{\prime}}\right) \frac{d r^{\prime}}{r^{\prime}}+\frac{2}{3} e_{1} r^{2}\right] \text {. }
$$

$$
\frac{1}{r^{3}} \int_{0}^{r}\left(4 \alpha^{\prime}-2 e^{\prime}-\frac{2}{3} r^{\prime} \frac{d e^{\prime}}{d r^{\prime}}\right) r^{4} d r^{\prime}=\frac{4}{3 r^{3}} \int_{0}^{r}\left(3 \alpha^{\prime}+e^{\prime}\right) r^{\prime 4} d r^{\prime}-\frac{2}{3} r^{2} e,
$$




$$
r^{2} \int_{r}^{a}\left(4 \alpha^{\prime}-2 e^{\prime}-\frac{2}{3} r^{\prime} \frac{d e^{\prime}}{d r^{\prime}}\right) \frac{d r^{\prime}}{r^{\prime}}=2 r^{2} \int_{r}^{a}\left(2 x^{\prime}-e^{\prime}\right) \frac{d r^{\prime}}{r^{\prime}}+\frac{2}{3} r^{2}\left(e-e_{1}\right),
$$

so that the expression for $V$ reduces to

$$
V=-\frac{2 g P_{2}}{5 a}\left[\frac{2}{r^{3}} \int_{0}^{r}\left(3 \alpha^{\prime}+e^{\prime}\right) r^{\prime 4} d r^{\prime}+3 r^{2} \int_{1}^{a}\left(2 \alpha^{\prime}-e^{\prime}\right) \frac{d r^{\prime}}{r^{\prime}}\right]
$$

The forces derived from the potential $V$ are (per unit mass)

$$
\begin{aligned}
& \frac{\partial V}{\partial r}=\frac{12 g P_{2}}{5 a}\left[\frac{1}{r^{4}} \int_{0}^{r}\left(3 \alpha^{\prime}+e^{\prime}\right) r^{4} d r^{\prime}-r \int_{r}^{a}\left(2 \alpha^{\prime}-e^{\prime}\right) \frac{d r^{\prime}}{r^{\prime}}-\frac{5}{6} e r\right], \\
& \frac{1}{r} \frac{\partial V}{\partial \theta}=\frac{3 g \sin 2 \theta}{5 a}\left[\frac{2}{r^{4}} \int_{0}^{r}\left(3 \alpha^{\prime}+e^{\prime}\right) r^{4} d r^{\prime}+3 r \int_{r}^{a}\left(2 \alpha^{\prime}-e^{\prime}\right) \frac{d r^{\prime}}{r^{\prime}}\right] .
\end{aligned}
$$

(4) Components of total bodily force.

Combining the foregoing results, we have for the components of the bodily force per unit volume at the point $(r, \theta)$,

$$
\begin{aligned}
\rho F_{r}=\frac{\rho g P_{2}}{a}\left[-\frac{2}{3} c r\right. & +\left(4 \alpha-4 e-\frac{2}{3} r \frac{d e}{d r}\right) r \\
& \left.+\frac{12}{5 r^{4}} \int_{10}^{r}\left(3 \alpha^{\prime}+e^{\prime}\right) r^{4} d r^{\prime}-\frac{12 r}{5} \int_{r}^{a}\left(2 \alpha^{\prime}-e^{\prime}\right) \frac{d r^{\prime}}{r^{\prime}}\right],
\end{aligned}
$$

$$
\rho F_{\theta}=\frac{\rho g \sin 2 \theta}{a}\left[\frac{1}{2} c r+\frac{6}{5 r^{4}} \int_{0}^{r}\left(3 \alpha^{\prime}+e^{\prime}\right) r^{\prime 4} d r^{\prime}+\frac{9 r}{5} \int_{r}^{a}\left(2 \alpha^{\prime}-e^{\prime}\right) \frac{d r^{\prime}}{r^{\prime}}\right]
$$

(e) Final form of equations of equilibrium for elementary volume. In writing the two equations of equilibrium for a volume element in their final form, it is convenient to introduce the factor $r$ throughout each equation, and then to replace $r$ as independent variable by $x=r / a$. The equations then take the following forms :

$$
\begin{aligned}
& 4(\lambda+\mu) x \frac{d \alpha}{d x}-8 \mu \alpha-\frac{2}{3}(\lambda+2 \mu) x^{2} \frac{d^{2} e}{d x^{2}}-\frac{8}{3}(\lambda+2 \mu) x \frac{d e}{d x}+4 \mu e \\
& +\rho g a\left[-\frac{2}{3} c x^{2}+\left(4 \alpha-4 e-\frac{2}{3} x \frac{d e}{d x}\right) x^{2}\right. \\
& \left.+\frac{12}{5 x^{3}} \int_{0}^{x}\left(3 \alpha^{\prime}+e^{\prime}\right) x^{4} d x^{\prime}-\frac{12 x^{2}}{5} \int_{x}^{1}\left(2 \alpha^{\prime}-e^{\prime}\right) \frac{d x^{\prime}}{x^{\prime}}\right]=0, \\
& \mu x^{2} \frac{d^{2} \alpha}{d x^{2}}+4 \mu x \frac{d \alpha}{d x}-2(3 \lambda+5 \mu) \alpha+(\lambda+\mu) x \frac{d e}{d x}+(3 \lambda+5 \mu) e \\
& +\rho g a\left[\frac{1}{2} c x^{2}+\frac{6}{5 x^{3}} \int_{0}^{x}\left(3 \alpha^{\prime}+e^{\prime}\right) x^{\prime \prime} d x^{\prime}+\frac{9 x^{2}}{5} \int_{x}^{1}\left(2 \alpha^{\prime}-e^{\prime}\right) \frac{d x^{\prime}}{x^{\prime}}\right]=0 \text {. }
\end{aligned}
$$




\section{§3. Surface conditions.}

Upon the bounding surface of the strained body the stress is zero. It seems most convenient, however, to treat the strained body as extending to or beyond

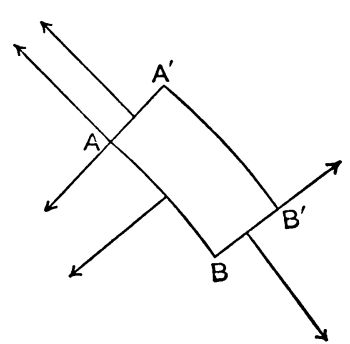

FIG. 1. the original spherical boundary $r=a$ in every direction, and to determine what stress must exist upon this surface in order that the surface $r=a\left(1-\frac{2}{3} e_{1} P_{2}\right)$ may be free from stress. For this purpose consider the layer of matter of density $\rho$ included between the surfaces $r=a, r=a\left(1-\frac{2}{3} e_{1} P_{2}\right)$. Let equations of equilibrium be formed for an elementary portion of this layer bounded by a meridian plane and a plane parallel to it at an infinitesimal distance $h$, and by two radial planes perpendicular to the meridian inclined to each other at an infinitesimal angle $\psi$; the section of such an element by the meridian plane is represented by $A B B^{\prime} A^{\prime}$ (Fig. 1).

We have

$$
\begin{gathered}
A B=a \psi, \quad A A^{\prime}=-\frac{2}{3} a e_{1} P_{2} \\
B B^{\prime}=A A^{\prime}+\psi \frac{\partial}{\partial \theta}\left(-\frac{2}{3} a e_{1} P_{2}\right)=A A^{\prime}+a e_{1} \psi \sin 2 \theta
\end{gathered}
$$

The stresses per unit area at the point $A$ are

$$
\begin{gathered}
\hat{r r}=\lambda \Delta+2 \mu \frac{\partial u_{r}}{\partial r}=\left[4 \lambda x-2\left(\lambda+\frac{2}{3} \mu\right) e-\frac{2}{3}(\lambda+2 \mu) x \frac{d e}{d x}\right] P_{2}, \\
\hat{\theta \theta}=\lambda \Delta+2 \mu\left(\frac{u_{r}}{r}+\frac{1}{r} \frac{\partial u_{\theta}}{\partial \theta}\right) \\
=\left[4 \lambda \alpha-2\left(\lambda+\frac{2}{3} \mu\right) e-\frac{2}{3} \lambda x \frac{d e}{d x}\right] P_{2}+4 \mu \alpha \cos 2 \theta \\
\hat{r} \hat{\theta}=\mu\left(\frac{\partial u_{\theta}}{\partial r}-\frac{u_{\theta}}{r}+\frac{1}{r} \frac{\partial u_{r}}{\partial \theta}\right)=\mu\left(e+x \frac{d \alpha}{d x}\right) \sin 2 \theta
\end{gathered}
$$

From these expressions values may be found for the total normal and tangential forces on the faces $A B, A A^{\prime}, B B^{\prime}$ of the element, while the total bodily force is the weight of the element, which is equal to $\rho g$ into its volume. By writing the two equations of equilibrium for the element, and retaining only terms of lowest order in the small quantities $e, \alpha$, it is found that

$$
\begin{gathered}
-\widehat{r r}+\rho g\left(\frac{2}{3} e a P_{2}\right)=0, \\
r \hat{\theta}=0 .
\end{gathered}
$$

The equations to be satisfied at the surface $r=a$ are therefore 


$$
\begin{gathered}
\widehat{\widehat{r r}}=4 \lambda \alpha-2\left(\lambda+\frac{2}{3} \mu\right) e-\frac{2}{3}(\lambda+2 \mu) x \frac{d e}{d x}=\frac{2}{3} \rho g a e, \\
\frac{\hat{P} \theta}{\sin 2 \theta}=\mu\left(e+x \frac{d \alpha}{d x}\right)=0 .
\end{gathered}
$$

\section{§4. Preliminary discussion of special cases.}

The problem is now reduced to that of solving the simultaneous ordinary differential equations (25) and (26), subject to the conditions expressed by (32) and (33). Before proceeding to the general solution, it is of interest to note the form assumed by the equations in special cases for which the solutions are known. Such cases are the following:

(A) A non-gravitating body acted upon by bodily disturbing forces having a potential proportional to $P_{2} r^{2}$ (or free from bodily forces), and

(1) With any given surface stresses of type corresponding to the bodily forces (i. e., the radial stress proportional to $P_{2}$ and the tangential stress to $\sin 2 \theta)$, or

(2) With any given surface displacements of the same type (i. e., the radial displacement proportional to $P_{2}$ and the tangential to $\sin 2 \theta$ ).

$(B) \mathrm{A}$ gravitating incompressible body with bodily and surface forces and surface displacements as in any of the cases under $(A)$.

Of these cases the complete solution will here be given only for that of a nongravitating body with surface free from stress. The modifications in the analysis required by the other cases under $(A)$ are sufficiently obvious, while the corresponding case of $(B)$ is given later on as a limiting case of the general solution for a gravitating compressible body.

It may be remarked, also, that after the general solution has been given it will be obvious how it must be modified if the surface conditions are changed.

(a) Solution for the case of a non-gravitating body acted upon by bodily forces but free from surface forces. In this case all the terms in equations (25) and (26) which represent bodily forces disappear except those representing the disturbing forces, and the equations become

$$
\begin{aligned}
& 4(\lambda+\mu) x \frac{d \alpha}{d x}-8 \mu \alpha-\frac{2}{3}(\lambda+2 \mu) x^{2} \frac{d^{2} e}{d x^{2}}-\frac{8}{3}(\lambda+2 \mu) x \frac{d e}{d x}+4 \mu e-\frac{2}{3} \rho g a c x^{2}=0 \\
& \mu x^{2} \frac{d^{2} \alpha}{d x^{2}}+4 \mu x \frac{d \alpha}{d x}-2(3 \lambda+5 \mu) \alpha+(\lambda+\mu) x \frac{d e}{d x}+(3 \lambda+5 \mu) e+\frac{1}{2} \rho g a c x^{2}=0
\end{aligned}
$$

The surface conditions (32) and (33) become

$$
4 \lambda \alpha-2\left(\lambda+\frac{2}{3} \mu\right) e-\frac{2}{3}(\lambda+2 \mu) x \frac{d e}{d x}=0, \quad e+x \frac{d \alpha}{d x}=0 \quad(x=1) .
$$


It is easily shown that these equations may all be satisfied by values of $e$ and $\alpha$ of the forms

$$
\begin{aligned}
& e=A_{0}+A_{2} x^{2}, \\
& \alpha=B_{0}+B_{2} x^{2},
\end{aligned}
$$

by properly determining the constants $A_{0}, A_{2}, B_{0}, B_{2}$.

To satisfy the differential equations we must have

$$
B_{0}=\frac{1}{2} A_{0},
$$

while $A_{2}$ and $B_{2}$ must satisfy the two equations

$$
\begin{aligned}
8 \lambda B_{2}-\frac{4}{3}(5 \lambda+7 \mu) A_{2}-\frac{2}{3} \rho g a c & =0, \\
-6 \lambda R_{2}+(5 \lambda+7 \mu) A_{2}+\frac{1}{2} \rho g a c & =0,
\end{aligned}
$$

which are seen to be identical. The surface conditions give two additional relations among the four constants; these are

$$
\begin{gathered}
4 \lambda\left(B_{0}+B_{2}\right)-2\left(\lambda+\frac{2}{3} \mu\right)\left(A_{0}+A_{2}\right)-\frac{4}{3}(\lambda+2 \mu) A_{2}=0, \\
A_{0}+A_{2}+2 B_{2}=0 .
\end{gathered}
$$

Solving these equations, we have

$$
\begin{array}{ll}
A_{0}=\frac{4 \lambda+3 \mu}{19 \lambda+14 \mu} \frac{\rho g a}{\mu} c, & A_{2}=-\frac{3 \lambda+2 \mu}{2(19 \lambda+14 \mu)} \frac{\rho g a}{\mu} c, \\
B_{0}=\frac{4 \lambda+3 \mu}{2(19 \lambda+14 \mu)} \frac{\rho g a}{\mu} c, & B_{2}=-\frac{5 \lambda+4 \mu}{4(19 \lambda+14 \mu)} \frac{\rho g a}{\mu} c .
\end{array}
$$

Hence, finally, the values

$$
\begin{aligned}
& e=\frac{c}{2(19 \lambda+14 \mu)}\left[2(4 \lambda+3 \mu)-(3 \lambda+2 \mu) x^{2}\right] \frac{\rho g a}{\mu}, \\
& \alpha=\frac{c}{4(19 \lambda+14 \mu)}\left[2(4 \lambda+3 \mu)-(5 \lambda+4 \mu) x^{2}\right] \frac{\rho g a}{\mu},
\end{aligned}
$$

which agree, with the known solution for this case.

(b) Non-gravitating body with given surface forces or surface displacements. If the two surface stresses are not zero but are known functions of $\theta$ of the type above specified, the last members of $(32)$ and (33) become known constants. If the surface displacements are given and of the type specified, (32) and (33) are replaced by equations obtained in an obvious manner.

(c) Non-gravitating spherical shell. The solution for a spherical shell may be obtained by assuming

$$
\begin{aligned}
& e=A_{-5} x^{-5}+A_{-3} x^{-3}+A_{0}+A_{2} x^{2}, \\
& \alpha=B_{-5} x^{-5}+B_{-3} x^{-3}+B_{0}+B_{2} x^{2},
\end{aligned}
$$


the constants being determined to satisfy the conditions at the inner and outer boundaries. It may in fact be shown that these equations satisfy (34) and (35) if $A_{0}, B_{0}, A_{2}, B_{2}$ satisfy (39) and (40), and the remaining coefficients the conditions

$$
\begin{gathered}
\mu A_{-3}-(3 \lambda+5 \mu) B_{-3}=0, \\
A_{-5}+3 B_{-5}=0 .
\end{gathered}
$$

There are thus four constants available for satisfying the boundary conditions.

(d) Incompressible gravitating body. In this case all the gravitational forces which are due to changes of density vanish, but the attraction of the surface inequality remains. The potential due to this surface layer has already been given, being

$$
\frac{8}{15} \pi \gamma \rho e_{1} r^{2} P_{2}=-\frac{2 g}{5 a} e_{1} r^{2} P_{2} .
$$

This gives in equations (25) and (26) the terms

$$
-\frac{4}{5} \text { pgae }_{1} x^{2} \text { and } \frac{3}{5} \text { pgae } x_{1}{ }^{2} \text {. }
$$

Since $e_{1}$ is a constant, the solution may proceed as in case $(a)$, by assuming

$$
\begin{aligned}
& e=A_{0}+A_{2} x^{2}, \\
& \alpha=B_{0}+B_{2} x^{2} .
\end{aligned}
$$

In satisfying the equations which determine the constants, special reasoning is required by the fact that $\lambda$ is infinite. This presents no serious difficulty, however, and will not be here given, since the case of incompressibility is hereinafter treated as a special case of the general problem of a gravitating sphere. To the solution of the general problem we now proceed.

\section{§5. Solution of equations in the general case.}

Inspection of the differential equations (25) and (26), and the form of the solution in the special cases above considered, suggest the trial of power series for the values of $e$ and $\alpha$.

The substitution of

$$
e=A_{m} x^{m}, \quad \alpha=B_{m} x^{m}
$$

results in the following terms in the two equations: In (25),

$$
\begin{gathered}
\left([4(\lambda+\mu) m-8 \mu] B_{m}-\left[\frac{2}{3}(\lambda+2 \mu)\left(m^{2}+3 m\right)-4 \mu\right] A_{m}\right) x^{m} \\
+\rho g a\left[\frac{2\left(m^{2}+8 m+6\right)}{3 m(m+5)}\left(6 B_{m}-(m+3) A_{m}\right) x^{m+2}\right. \\
\left.-\frac{12}{5 m}\left(2 B_{m}-A_{m}\right) x^{2}\right]
\end{gathered}
$$


In (26),

$$
\begin{gathered}
\left(\left[\mu\left(m^{2}+3 m\right)-2(3 \lambda+5 \mu)\right] B_{m}+[(\lambda+\mu) m+(3 \lambda+5 \mu)] A_{m}\right) x^{m} \\
+\rho g a\left[-\frac{3}{m(m+5)}\left(6 B_{m}-(m+3) A_{m}\right) x^{m+2}\right. \\
\left.+\frac{9}{5 m}\left(2 B_{m}-A_{m}\right) x^{2}\right] .
\end{gathered}
$$

Notice first that the coefficients of $x^{2}$ in these two expressions are in a constant ratio independent of $m$; hence if $m$ be given a series of values, the coefficients of $x^{2}$ in the two equations may be made to vanish by a single relation among the coefficients.

Again, notice that terms in $x^{m+2}$ result only from the following terms in the values of $e$ and $\alpha$ :

$$
A_{m} x^{m}+A_{m+2} x^{m+2}, \quad B_{m} x^{n}+B_{m+2} x^{m+2} ;
$$

and that the coefficients of $x^{m+2}$ in both equations will vanish if the following two equations are satisfied:

$$
\begin{aligned}
{[4(\lambda+\mu) m+8 \lambda] B_{m+2}-} & {\left[\frac{2}{3}(\lambda+2 \mu) m(m+7)+\frac{4}{3}(5 \lambda+7 \mu)\right] A_{m+2} } \\
+\rho g a & \frac{2\left(m^{2}+8 m+6\right)}{3 m(m+5)}\left[6 B_{m}-(m+3) A_{m}\right]=0, \\
{[\mu m(m+7)-6 \lambda] B_{m+2}+} & {[(\lambda+\mu) m+5 \lambda+7 \mu] A_{m+2} } \\
& -\rho g a \frac{3}{m(m+5)}\left[6 B_{m}-(m+3) A_{m}\right]=0 .
\end{aligned}
$$

Fipally, notice that the terms of lowest degree in the series for $e$ and $\alpha$ will introduce into the two equations terms of like degree which must vanish identically. That is, the terms

$$
A_{m} x^{m}, B_{m} x^{m}
$$

of lowest degree must be such that the coefficients of $x^{m}$ in (52) and (53) will vanish. The values of $m$ for which this is possible without the vanishing of $A_{m}$ and $B_{m}$ are found by equating to zero the determinant of the coefficients of $A_{m}$ and $B_{m}$ in these expressions; this determinant reduces to

$$
\frac{2}{3} \mu(\lambda+2 \mu)(m-2) m(m+3)(m+5) .
$$

The lowest power of $x$ in the two series must therefore be either

$$
x^{2}, x^{0}, x^{-3} \text { or } x^{-5} \text {. }
$$

Since negative powers of $x$ are excluded by physical considerations (because the solution must apply to the point $x=0$ ), we conclude that the terms available 
for the solution constitute ascending series of even powers of $x$ beginning with the power zero. It is easily found also that we must have ${ }^{*} B_{0}=\frac{1}{2} A_{0}$.

We now assume

$$
\begin{aligned}
& e=A_{0}+A_{2} x^{2}+A_{4} x^{4}+\cdots, \\
& \alpha=\frac{1}{2} A_{0}+B_{2} x^{2}+B_{4} x^{4}+\cdots,
\end{aligned}
$$

and determine the relations which must hold among the coefficients in order that the coefficient of every power of $x$ in each of two equations may vanish identically.

From the above discussion it is evident that if $m>0$, the equations to be satisfied are (54) and (55), while the vanishing of the coefficient of $x^{2}$ requires that

$$
8 \lambda B_{2}-\frac{4}{3}(5 \lambda+7 \mu) A_{2}-g \rho a\left[\frac{4}{5} A_{0}+\frac{12}{5} \sum_{m=2}^{\infty} \frac{1}{m}\left(2 B_{m}-A_{m}\right)\right]=\frac{2}{3} \rho g a c
$$

(odd values of $m$ being excluded in the summation).

The solution of equations (54) and (55) gives

$$
\begin{aligned}
& A_{m+2}=\frac{\mu m^{2}+10 \mu m-6(\lambda-2 \mu)}{\mu m(m+2)(m+5)(m+7)}\left[6 B_{m}-(m+3) A_{m}\right] \frac{g a \rho}{\lambda+2 \mu}, \\
& B_{m+2}=-\frac{(\lambda+\mu) m+5 \lambda+4 \mu}{\mu m(m+2)(m+5)(m+7)}\left[6 B_{m}-(m+3) A_{m}\right] \frac{g a \rho}{\lambda+2 \mu}, \\
& 6 B_{m+2}-(m+5) A_{m+2}=-\frac{m+6}{m(m+5)}\left[6 B_{m}-(m+3) A_{m}\right] \begin{array}{c}
g a \rho \\
\lambda+2 \mu
\end{array}
\end{aligned}
$$

By successive applications of the last equation may be derived the following values of the coefficients of $x^{4}$ and higher powers, $n$ having values $0,1,2, \ldots$ :

$$
\begin{aligned}
& \left.(62) A_{4+2 n}=-{ }_{24}^{1}\left(6 B_{2}-5 A_{2}\right)\left(-\frac{g a \rho}{\lambda+2 \mu}\right)^{n+1}\left[4\left(n^{2}+7 n+9\right)-6 \frac{{ }^{\lambda}}{\mu}\right] \frac{n+3}{7 \cdot 9 \cdots(2 n+9}\right)^{\prime} \\
& \text { (63) } B_{t+2 n}={ }_{24}^{1}\left(6 B_{2}-5 A_{2}\right)\left(-\frac{g a \rho}{\lambda+2 \mu}\right)^{n+1}\left[2(n+3)+(2 n+7)_{\mu}^{\lambda}\right] \frac{n+3}{7 \cdot 9 \cdots(2 n+9)^{\prime}}
\end{aligned}
$$

so that the values of $e$ and $\alpha$ may be written

$$
\begin{aligned}
e= & A_{0}+A_{2} x^{2}+{ }_{2 \pm}^{1}\left(6 B_{2}-5 A_{2}\right) \frac{g a \rho}{\lambda+2 \mu} x^{4} \\
& \times \sum_{0}^{\infty}(-1)^{n}\left[4\left(n^{2}+7 n+9\right)-6 \begin{array}{c}
\lambda \\
\mu
\end{array}\right] \frac{n+3}{7 \cdot 9 \cdots(2 n+9)}\left(\frac{g c \rho}{\lambda+2 \mu} x^{2}\right)^{n},
\end{aligned}
$$

* This is evident algebraically. It is also evident otherwise that if the solution has the assumed form, $B_{0}$ must equal ${ }_{2}^{1} A_{0}$; for $A_{0}$ and $B_{0}$ are the values of $e$ and $\alpha$ at the center, where these quantities will be related as in the case of a homogeneous strain. 


$$
\begin{aligned}
\alpha= & \frac{1}{2} A_{0}+B_{2} x^{2}-\frac{1}{24}\left(6 B_{2}-5 A_{2}\right) \frac{g a \rho}{\lambda+2 \mu} x^{4} \\
& \times \sum_{0}^{\infty}(-1)^{n}\left[2(n+3)+(2 n+7) \frac{\lambda}{\mu}\right] \frac{n+3}{7 \cdot 9 \cdots(2 n+9)}\left(\frac{g a \rho}{\lambda+2 \mu} x^{2}\right)^{n},
\end{aligned}
$$

in which $A_{0}, A_{2}, B_{2}$ must satisfy (58) and the surface conditions (32) and (33).

For substitution in (58) we find (for $n=0,1,2, \ldots$ )

$$
\begin{aligned}
\frac{2 B_{4+2 n}-A_{4+2 n}}{4+2 n}=\frac{1}{12}\left(6 B_{2}-5 A_{2}\right) & \left(-\frac{g \alpha \rho}{\lambda+2 \mu}\right)^{n+1} \\
& \times\left(n+6+\frac{\lambda}{\mu}\right) \frac{n+3}{7 \cdot 9 \cdots(2 n+9)} .
\end{aligned}
$$

In order to express as concisely as possible the values of $e$ and $\alpha$ and the three equations to determine the constants, the following notation may be employed :

$$
\begin{aligned}
L(z) & =\sum_{0}^{\infty}(-1)^{n}\left[4\left(n^{2}+7 n+9\right)-6 \frac{\lambda}{\mu}\right] \frac{n+3}{7 \cdot 9 \cdots(2 n+9)} z^{n}, \\
M(z) & =\sum_{0}^{\infty}(-1)^{n}\left[2(n+3)+(2 n+7) \frac{\lambda}{\mu}\right] \frac{n+3}{7 \cdot 9 \cdots(2 n+9)} z^{n}, \\
N(z) & =\sum_{0}^{\infty}(-1)^{n}\left[n+6+\frac{\lambda}{\mu}\right] \frac{n+3}{7 \cdot 9 \cdots(2 n+9)} z^{n}, \\
L^{\prime}(z) & =\sum_{0}^{\infty}(-1)^{n}\left[4\left(n^{2}+7 n+9\right)-6 \frac{\lambda}{\mu}\right] \frac{(n+2)(n+3)}{7 \cdot 9 \cdots(2 n+9)} z^{n}, \\
M^{\prime}(z) & =\sum_{0}^{\infty}(-1)^{n}\left[2(n+3)+(2 n+7) \frac{\lambda}{\mu}\right] \frac{(n+2)(n+3)}{7 \cdot 9 \cdots(2 n+9)} z^{n}, \\
b & =\frac{g a \rho}{\lambda+2 \mu} .
\end{aligned}
$$

The solution then takes the following form, the last two of the five equations being derived from the surface conditions (32) and (33):

$$
\begin{gathered}
e=A_{0}+A_{2} x^{2}+\frac{1}{24}\left(6 B_{2}-5 A_{2}\right) b x^{4} L\left(b x^{2}\right), \\
\alpha=\frac{1}{2} A_{0}+B_{2} x^{2}-\frac{1}{2} 4\left(6 B_{2}-5 A_{2}\right) b x^{4} M\left(b x^{2}\right), \\
4\left(A_{0}+A_{2}\right)+\frac{140}{3 b(\lambda / \mu+2)} A_{2} \\
+\left(6 B_{2}-5 A_{2}\right)\left(2-\frac{20 \lambda / \mu}{3 b(\lambda / \mu+2)}-b N(b)\right)+\frac{10}{3} c=0,
\end{gathered}
$$


(D)

$$
\begin{aligned}
& {\left[2+\left(\frac{\lambda}{\mu}+2\right) b\right]\left(A_{0}+A_{2}\right)+4 A_{2}-\left(6 B_{2}-5 A_{2}\right)} \\
& \times\left\{\frac{\lambda}{\mu}-\frac{b}{24}\left[\left(3 \frac{\lambda}{\mu}+2+\left(\frac{\lambda}{\mu}+2\right) b\right) L(b)+6 \frac{\lambda}{\mu} M(b)+2\left(\frac{\lambda}{\mu}+2\right) L^{\prime}(b)\right]\right\}=0,
\end{aligned}
$$

$$
3\left(A_{0}+A_{2}\right)+5 A_{2}+\left(6 B_{2}-5 A_{2}\right)\left[1-\frac{b}{8}\left[L(b)-2 M^{\prime}(b)\right]\right]=0 \text {. }
$$

The functions represented by the infinite series may be otherwise expressed, depending ultimately upon the exponential integral

$$
\int_{0}^{x} e^{16 x^{2}} d x
$$

For the purposes of computation, however, it is doubtful whether they can be reduced to expressions more convenient than the series, which converge with satisfactory rapidity unless $b$ is relatively large. In applying the results to a body comparable in size and mass with the earth, for example, it can hardly be supposed that $b$ is as great as 2 ; and for $b=2$ it is found that eight or ten terms of the series suffice, even in case of the function $L^{\prime}$, which is the least rapidly convergent of all.

\section{$\S 6$. Modified procedure for solving equations.}

It may be well to point ont that by changing the procedure the algebraic work involved in the solution of equations (25) and (26) can be somewhat abridged.

The equations of equilibrium of a volume element may be abbreviated by introducing the elementary rotations $\varpi_{r}, \varpi_{\theta}, \varpi_{\phi}$. The general forms of these equations are given in Part IV of this paper, equations (117), ., (123). When the strain is symmetrical about the initial line of the system of coordinates, $\varpi_{r}$ and $\varpi_{\theta}$ vanish, and the equations reduce to the two following, which are identical with (1) and (2);

$$
\begin{gathered}
(\lambda+2 \mu) \frac{\partial \Delta}{\partial r}-\frac{2 \mu}{r \cdot \sin \theta} \frac{\partial}{\partial \theta}\left(\varpi_{\theta} \sin \theta\right)+\rho F_{r}=0, \\
(\lambda+2 \mu) \frac{1}{r} \frac{\partial \Delta}{\partial \theta}+r_{r} \sin \theta \frac{\partial}{\partial r}\left(r \varpi_{\phi} \sin \theta\right)+\rho F_{\theta}=0 .
\end{gathered}
$$

If we now write

$$
\Delta=y P_{2}, \quad 2 \varpi_{\phi}=z \frac{\partial P_{2}}{\partial \theta},
$$


and introduce everywhere $y$ and $z$ instead of $e$ and $\alpha$, equations (25) and (26) become

$$
\begin{aligned}
& (\lambda+2 \mu) x \frac{d y}{d x}+6 \mu z \\
& +\rho g a\left[\frac{9}{5 x^{3}} \int_{0}^{x} y^{\prime} x^{\prime 4} d x^{\prime}-\frac{6 x^{2}}{5} \int_{x}^{1} \frac{y^{\prime} d x^{\prime}}{x^{\prime}}+y x^{2}-\left(\frac{4}{5} e_{1}+\frac{2}{3} c\right) x^{2}\right]=0, \\
& (\lambda+2 \mu) y+\mu \frac{d(x z)}{d x} \\
& \quad-\rho g a\left[\frac{3}{5 x^{3}} \int_{0}^{x} y^{\prime} x^{\prime 4} d x^{\prime}+\frac{3 x^{2}}{5} \int_{x}^{1} \frac{y^{\prime} d x^{\prime}}{x^{\prime}}+\left(\frac{2}{5} e_{1}+\frac{1}{3} c\right) x^{2}\right]=0 .
\end{aligned}
$$

It is easily shown that these are satisfied by series of the following forms :

$$
y=C_{2} x^{2}+C_{4} x^{4}+\cdots, \quad z=D_{2} x^{2}+D_{4} x^{4}+\cdots,
$$

in which the coefficients satisfy the equations

$$
\begin{aligned}
\frac{C_{m+2}}{C_{m}^{-}} & =-\frac{m+6}{m(m+5)} \cdot \frac{\rho g a}{\lambda+2 \mu}, \\
\frac{D_{m+2}}{C_{m}^{-}} & =\frac{1}{m(m+5)} \cdot \frac{\rho g a}{\mu},
\end{aligned}
$$

and an additional relation obtained by equating to 0 the coefficients of $x^{2}$ after substituting the values of $y$ and $z$ in the differential equations. One coefficient thus remains arbitrary.*

It is now possible to determine $e$ and $\alpha$ from the equations

Thus if we assume

$$
4 \alpha-2 e-\frac{2}{3} x \frac{d e}{d x}=y, \quad \frac{2}{3}\left(e-2 \alpha-x \frac{d \alpha}{d x}\right)=z .
$$

we find

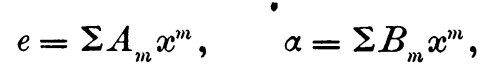

$$
\begin{gathered}
(m+3) A_{m}-6 B_{m}=-\frac{3}{2} C_{m}, \\
A_{m}-(m+2) B_{m}=\frac{3}{2} D_{m},
\end{gathered}
$$

from which $A_{m}$ and $B_{m}$ are determined in terms of $C_{m}$ and $D_{m}$ (and therefore in terms of the one arbitrary constant above mentioned), except in the case $\dagger$ $m=0$. In this case we may take $A_{0}$ arbitrarily and make

$$
B_{0}=\frac{1}{2} A_{0} \text {. }
$$

\footnotetext{
* Another solution consists of ascending series beginning with $C_{-3} x^{-3}$ and $D_{-3} x^{-3}$; but negative powers of $x$ are not permissible in the physical problem.

$\dagger$ The case $m=-5$ is excluded by physical considerations.
} 
That is, the values of $e$ and $\alpha$ contain the same powers of $x$ as the values of $y$ and $z$, together with the absolute terms $A_{0}$ and $\frac{1}{2} A_{0}$. Two arbitrary constants remain for satisfying the surface conditions.

Although this process results in some saving of algebraic work, it does not appear to suggest any shortening of the labor involved in numerical computations such as those given below.

It is of some interest to note the form of the differential equation resulting from the elimination of $z$ :

$$
x^{2} \frac{d^{2} y}{d x^{2}}+2 x \frac{d y}{d ! c}-6 y+\frac{\rho g a}{\lambda+2 \mu}\left(x^{3} \frac{d y}{d x}+6 x^{2} y\right)=0 .
$$

We obtain also the equation

$$
x^{2} \frac{d^{2} z}{d x^{2}}+2 x \frac{d z}{d x}-6 z-\frac{\rho g a}{\mu} x^{2} y=0 ;
$$

but the complete elimination of $y$ obviously leads to an equation of the fourth order in $z$.

\section{§ 7. External potential and inertia-constant.}

One of the quantities which indicates the yielding of the body is the change in the external potential, or (what amounts to the same thing) in the difference between the principal moments of inertia.

Let $I$ denote the moment of inertia about a diameter before strain, $C$ and $A$ the principal moments after strain (the former being taken about the axis of symmetry), and let

$$
f=\frac{C}{I}-\frac{A}{}
$$

The value contributed to $C-A$ by an elementary shell of thickness $d r$ and density

is

$$
-\rho \Delta=-\rho\left(4 \alpha-2 e-\frac{2}{3} r \frac{d e}{d r}\right) P_{2}
$$

$$
\frac{4}{5} \pi r^{4} \rho\left(4 \alpha-2 e-\frac{2}{3} r \frac{d e}{d r}\right) d r,
$$

while the part contributed by the surface inequality is

hence

$$
\frac{8}{15} \pi \rho a^{5} e_{1}
$$

$$
C-A=\frac{4 \pi \rho}{5} \int_{0}^{\prime \prime}\left(4 x-2 e-\frac{2}{3} r \frac{d e}{d r}\right) r^{4} d r+\frac{8}{15} \pi \rho a^{5} e_{1} .
$$

Integrating by parts the term containing $d e / d r$, we obtain 


$$
C-A=\frac{16 \pi \rho}{15} \int_{0}^{a}(3 \alpha+e) r^{4} d r=\frac{16 \pi \rho a^{5}}{15} \int_{0}^{1}(3 \alpha+e) x^{4} d x
$$

and

$$
f=\frac{C-A}{I}=2 \int_{0}^{1}(3 \alpha+e) x^{4} d x .
$$

This accords with the result already given for the potential due to the change of configuration. Thus the external potential is known to have the value

$$
-\frac{\gamma(C-A)}{r^{3}} P_{2}
$$

while it is also represented by the first term of the value of $V$ given in (20) if the upper limit of integration be $a$; i. e., we must have

$-\frac{\gamma(C-A)}{r^{3}} P_{2}=-\frac{4 g P_{2}}{5 a r^{3}} \int_{0}^{a}\left(3 \alpha^{\prime}+e^{\prime}\right) r^{\prime 4} d r^{\prime}=-\frac{16 \pi \rho \gamma P_{2}}{15 r^{3}} \int_{0}^{a}(3 \alpha+e) r^{4} d r$,

which agrees with the result given above.

Using the values above found for $e$ and $\alpha$, we may write

in which

$$
f=\frac{C-A}{I}=A_{0}+A_{2}+\left(6 B_{2}-5 A_{2}\right)\left[\frac{1}{7}+\frac{b}{12}\left(2 G(b)-3 \frac{\lambda}{\mu} F(b)\right)\right] .
$$

$$
\begin{aligned}
& F(b)=\sum_{0}^{\infty}(-1)^{n} \frac{n+3}{7 \cdot 9 \cdots(2 n+9)} b^{n}, \\
& G(b)=\sum_{0}^{\infty}(-1)^{n} \frac{(n+1)(n+3)}{7 \cdot 9 \cdots(2 n+9)} b^{n} .
\end{aligned}
$$

\$8. Case of incompressibility.

In the case of incompressibility $\lambda=\infty, b=0$. Putting $b^{\prime}=g a \rho / \mu$, we find

$$
\begin{aligned}
& b L(b)=\frac{g a \rho}{\lambda+2 \mu}\left(-6 \frac{\lambda}{\mu}\right) \frac{3}{7 \cdot 9}=-\frac{2}{7} b^{\prime}, \\
& b M(b)=\frac{g a \rho}{\lambda+2 \mu}\left(7 \frac{\lambda}{\mu}\right) \frac{3}{7 \cdot 9}=\frac{1}{3} b^{\prime}, \\
& L N(b)=\frac{g a \rho}{\lambda+2 \mu}\left(\frac{\lambda}{\mu}\right) \frac{3}{7} \frac{9}{9}=\frac{1}{2} b^{\prime}, \\
& b L^{\prime}(b)=\frac{g a \rho}{\lambda+2 \mu}\left(-6 \frac{\lambda}{\mu}\right)_{7 \cdot \frac{6}{9}}=-\frac{4}{7} b^{\prime}, \\
& b M^{\prime}(b)=\frac{g a \rho}{\lambda+2 \mu}\left(7 \frac{\lambda}{\mu}\right) \frac{6}{7 \cdot 9}=\frac{2}{3} b^{\prime} .
\end{aligned}
$$


Equation $(C)$ now requires that

$$
\begin{gathered}
6 B_{2}-5 A_{2}=0 \\
\left(6 B_{2}-5 A_{2}\right) \frac{\lambda}{\mu}=\frac{3 b^{\prime}}{20}\left[4 A_{0}+4\left(1+\frac{35}{3 b^{\prime}}\right) A_{2}+\frac{1 Q}{3} C\right] .
\end{gathered}
$$

while $(D)$ and $(E)$ give

$$
\begin{gathered}
\left(6 B_{2}-5 A_{2}\right) \frac{\lambda}{\mu}=\left(2+b^{\prime}\right) A_{0}+\left(6+b^{\prime}\right) A_{2}, \\
3 A_{0}+8 A_{2}=0 .
\end{gathered}
$$

Solving, we obtain

$$
A_{0}=\frac{8 b^{\prime}}{38+4 b^{\prime}} c, \quad A_{2}=-\frac{3 b^{\prime}}{38+4 b^{\prime}} c, \quad B_{2}=-\frac{5 b^{\prime}}{2\left(38+4 b^{\prime}\right.} c,
$$

while all other coefficients in the values of $e$ and $\alpha$ vanish. We thus have

$$
\begin{gathered}
\frac{e}{c}=\frac{b^{\prime}}{38+4 b^{\prime}}\left(8-3 x^{2}\right), \\
\frac{\alpha}{c}=\frac{b^{\prime}}{2\left(38+4 b^{\prime}\right)}\left(8-5 x^{2}\right),
\end{gathered}
$$

which agree with the known solution for the case of incompressibility.

We find also

$$
f=A_{0}+A_{2}=\frac{5 b^{\prime} c}{38+4 b^{\prime}},
$$

which is identical with the surface value of $e$, as should be the case when $\lambda=\infty$.

\section{§9. Case of zero rigidity.}

This is the only case in which the solution fails. If $\mu=0$, equations (25) and (26) cannot be satisfied unless $\Delta=0$; and if $\Delta=0$, the surface condition (32) cannot be satisfied unless $\lambda=\infty$.

\section{$\S 10$. Numerical results.}

In applying the formulas above fcund, it is convenient to choose a definite value of $\lambda / \mu$ and make computations for a series of values of $b$ or $g \rho a /(\lambda+2 \mu)$. Some of the results thus found are given in the accompanying tables and diagrams, ${ }^{*}$ which for the most part are self-explanatory. The quantities of particular interest are the surface values of $e$ and $\alpha$ and $f$. As an aid to the

\footnotetext{
* In all the diagrams it is to be understood that ordinates are multiplied by $c$.
} 
verification of the computations, the values of the functions $L, M$, etc., are included in the tables.

TABLE I. Results for the case $\lambda / \mu=1$.

(All numbers below the horizontal line are to be multiplied by $c$. Numbers in square brackets [ ] obtained by interpolation ; all others computed.)

\begin{tabular}{c|c|c|c|c|c|c|c|c}
\hline$b=\frac{g a \rho}{\lambda+2 \mu}$ & 2 & 1.8 & 1.6 & 1.4 & 1.2 & 1 & .8 & .667 \\
\hline$\mu / g a \rho$ & .167 & .185 & .208 & .238 & .278 & .333 & .417 & .500 \\
$L(b)$ & .8946 & .9347 & .9773 & 1.0224 & 1.0703 & 1.1212 & 1.1753 & 1.2133 \\
$M(b)$ & .4618 & .4746 & .4879 & .5018 & .5163 & .5315 & .5474 & .5585 \\
$N(b)$ & .2579 & .2641 & .2706 & .2774 & .2844 & .2917 & .2994 & .3026 \\
$L !(b)$ & 1.4000 & 1.4978 & 1.6042 & 1.7194 & 1.8444 & 1.9801 & 2.1273 & 2.2326 \\
$M^{\prime}(b)$ & .7987 & .8319 & .8668 & .9039 & .9434 & .9853 & 1.0298 & 1.0610 \\
$F(b)$ & .03798 & .03880 & .03964 & .04052 & .04142 & .04236 & .04333 & .04400 \\
$G(b)$ & .02999 & {$[.031]$} & {$[.033]$} & {$[.034]$} & {$[.036]$} & .03758 & .03936 & .04061 \\
\hline $6 B_{2}-5 A_{2}$ & 3.710 & 2.857 & 2.141 & 1.552 & 1.067 & .7004 & .4152 & .2701 \\
$A_{0}=e_{0}$ & .5146 & .5251 & .5218 & .5058 & .4758 & .4327 & .3760 & .3303 \\
$A_{2}$ & -.5752 & -.4954 & -.4228 & -.3570 & -.2964 & -.2405 &.- .1883 & -.1551 \\
$B_{2}$ & .139 & .0633 & .0045 & -.0388 & -.0677 & -.0837 & -.0877 & -.0842 \\
$e_{1}$ & .216 & .230 & .238 & .241 & .237 & .225 & .204 & .1843 \\
$f$ & .436 & .415 & .390 & .361 & .327 & .289 & .246 & .213 \\
$\alpha_{1}$ & .253 & .224 & .196 & .169 & .142 & .117 & .0927 & .0767 \\
\hline
\end{tabular}

TABLE II. Results for the case $\lambda / \mu=2$.

(Numbers below the horizontal line to be multiplied by $e$. Numbers in square brackets obtained by interpolation ; all others computed.)

\begin{tabular}{|c|c|c|c|c|c|c|c|c|}
\hline$b=\frac{g a \rho}{\lambda+2 \mu}$ & 2 & 1.8 & 1.6 & 1.4 & 1.2 & 1 & .8 & .667 \\
\hline$\mu / g a \rho$ & .125 & .139 & .156 & .179 & .208 & .250 & .3125 & .375 \\
\hline$L(b)$ & .6667 & .7019 & .7394 & .7793 & .8218 & .8671 & .9153 & .9493 \\
\hline$M(b)$ & .7117 & .7312 & .7516 & .7729 & .7952 & .8185 & .8428 & .8597 \\
\hline$N(b)$ & .2959 & .3029 & .3103 & .3179 & .3258 & .3341 & .3427 & .3467 \\
\hline$L^{\prime}(b)$ & .9922 & 1.0771 & 1.1697 & 1.2706 & 1.3806 & 1.5005 & 1.6311 & 1.7367 \\
\hline$M^{\prime}(b)$ & 1.2321 & 1.2829 & 1.3365 & 1.3933 & 1.4537 & 1.5179 & 1.5860 & 1.6328 \\
\hline$F(b)$ & .03798 & [.0388] & [.0396] & [.0405] & [.0414] & .04236 & .04333 & .04400 \\
\hline$G(b)$ & .02999 & {$[.031]$} & {$[.033]$} & {$[.034]$} & {$[.036]$} & .03758 & .03936 & .04061 \\
\hline $6 B_{2}-5 A_{2}$ & 3.749 & 2.915 & 2.209 & 1.623 & 1.142 & .7577 & .4596 & .3056 \\
\hline$A_{0}=e_{0}$ & .5537 & .5852 & .5984 & .5935 & .5698 & .528 & .4666 & .4144 \\
\hline$A_{2}$ & -.4655 & -.4309 & -.3938 & -.3542 & -.3118 & -.267 & -.2195 & -.1862 \\
\hline$B_{2}$ & .2369 & .1267 & .0400 & -.0247 & -.0695 & -.0962 & -.1063 & -.1042 \\
\hline$e_{1}$ & .2964 & .3078 & .3135 & .3131 & .3049 & .289 & .2611 & .236 \\
\hline$f$ & .519 & .496 & .469 & .438 & .401 & .358 & .307 & .269 \\
\hline$\alpha_{1}$ & .2914 & .259 & $.2: 8$ & .199 & .170 & .142 & .1140 & .096 \\
\hline
\end{tabular}


TABLE III. Results for the case $\lambda / \mu=3$.

(Numbers below the horizontal line to be multiplied by $c$. Numbers in square brackets obtained by interpolation ; all others computed.)

\begin{tabular}{|c|c|c|c|c|c|c|c|c|}
\hline$b=\frac{g a \rho}{\lambda+2 \mu}$ & 2 & 1.8 & 1.6 & 1.4 & 1.2 & 1 & .8 & .667 \\
\hline$\mu / g a \rho$ & .10 & .111 & .125 & .143 & .167 & .200 & .250 & .300 \\
\hline$L(b)$ & .4388 & .4691 & .5016 & .5362 & .5733 & .6129 & .6553 & .6853 \\
\hline$M(b)$ & .9616 & .9880 & 1.0154 & 1.0441 & 1.0740 & 1.1054 & 1.1381 & 1.1610 \\
\hline$N(b)$ & .3329 & .3417 & .3499 & .3584 & .3672 & .3764 & .3861 & .3906 \\
\hline$L^{\prime}(b)$ & .5840 & .6566 & .7354 & .8218 & .9168 & 1.0212 & 1.1349 & 1.2414 \\
\hline$M^{\prime}(b)$ & 1.6654 & 1.7339 & 1.8060 & 1.8826 & 1.9641 & 2.0505 & 2.1423 & 2.2046 \\
\hline$F(b)$ & .03798 & .03880 & .03964 & .04052 & .04142 & .04236 & .04333 & .04400 \\
\hline$G(b)$ & .02999 & {$[.031]$} & {$[.033]$} & {$[.034]$} & {$[.036]$} & .03758 & .03936 & .04061 \\
\hline $6 B_{2}-5 A_{2}$ & 3.910 & 3.032 & 2.320 & 1.721 & 1.224 & .823 & .497 & .343 \\
\hline$A_{0}=e_{0}$ & .545 & .598 & .643 & .654 & .642 & .605 & .532 & .489 \\
\hline$A_{2}$ & -.340 & -.348 & -.351 & -.339 & -.317 & -.285 & -.239 & -.214 \\
\hline$B_{2}$ & .368 & .215 & .094 & .0043 & -.0602 & -.1003 & -.1163 & -.1215 \\
\hline$e_{1}$ & .3487 & {$[.365]$} & .370 & .369 & .360 & .341 & {$[.314]$} & .281 \\
\hline$f$ & .580 & .556 & .533 & .501 & .463 & .417 & {$[.370]$} & .318 \\
\hline$a_{1}$ & .327 & .290 & .258 & .226 & .195 & .164 & .131 & .112 \\
\hline
\end{tabular}

In Figs. 2 and 3 are shown curves representing $e_{1}, \alpha_{1}$ and $f$ as functions of $\mu / g \rho a$, for certain constant values of $\lambda / \mu$. It is seen that, as $\lambda / \mu$ increases, the curves for $e_{1}$ and $f$ both approach the curve $(A)$ which represents the case $\lambda=\infty$. For a given value of $\mu$ both $e_{1}$ and $f$ increase with $\lambda$; in other words, the effect of compressibility is to decrease both the ellipticity and the external potential corresponding to a certain rigidity. This effect is much more important upon $e_{1}$ than upon $f$. Thus if $\mu=.35 \rho g a$, a variation of $\lambda / \mu$ from 1 to $\infty$ would change $f / c$ only from .279 to .289 , while $e_{1} / c$ would range from .221 to .289 . For smaller values of $\mu$ the effect of compressibility is greater. An interesting feature is the fact that for a given value of $\lambda / \mu$ there is a maximum value of $e_{1}$; thus if $\lambda=\mu$, a decrease of $\mu$ below about .24 $\rho g a$ causes $e_{1}$ to decrease.

Fig. 3 shows also that the value of $\alpha_{1}$ is increased to an important extent by compressibility.

\section{Strain of an elastic sphere covered by a shallow ocean.}

\section{§1. Statement of problem.}

In applying the theory of the deformation of a sphere to the case of the earth, an important question is the effect of the ocean. Some light on the order of magnitude of this effect is thrown by the solution of the following problem:

To determine the strain of a gravitating elastic sphere, the surface of which is covered by a shallow ocean in equilibrium.

The solution above given may without difficulty be extended to this case, as follows. 
§2. Forces due to ocean inequality.

Let $c^{\prime} \rho=$ density of ocean,

$e=$ ellipticity of ocean surface.

Then there must be introduced into the solution the forces due to an ocean layer of thickness

$$
-\frac{2}{3}\left(\mathrm{e}-e_{1}\right) a P_{2} .
$$

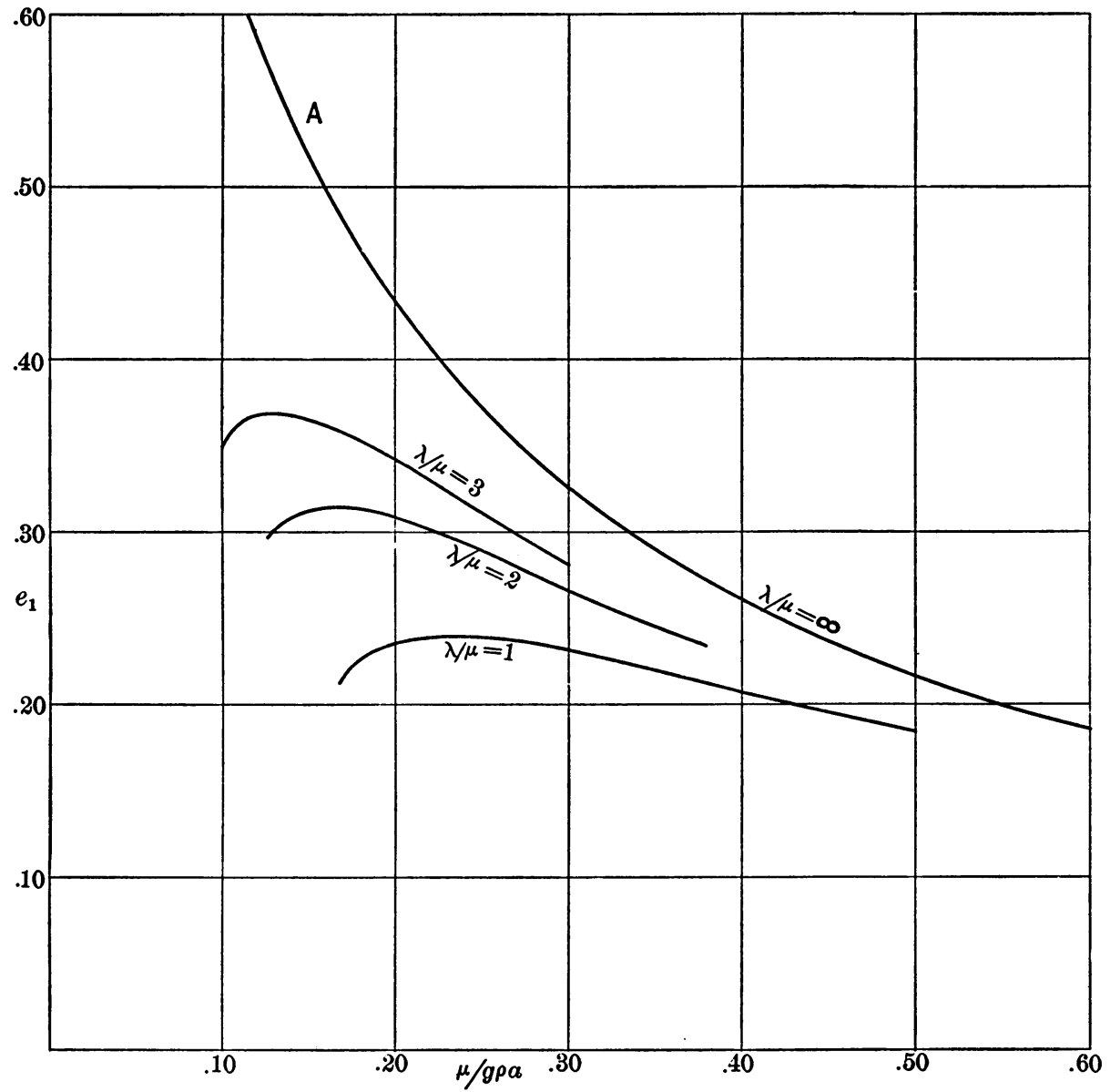

Fra. 2. Curves showing effect of compressibility upon surface ellipticity.

These forces will be $(a)$ the bodily force due to the attraction of the layer, and (b) the surface pressure due to the weight of the layer.

(a) The attraction is given by the potential

$$
V^{\prime}=-{ }_{15}^{8} \pi \gamma c^{\prime} \rho\left(\mathrm{e}-e_{1}\right) r^{2} P_{2}=-\frac{2 g}{5 a} c^{\prime}\left(\mathrm{e}-e_{1}\right) r^{2} P_{2},
$$


which adds to (23) and (24) the terms

$$
\begin{gathered}
\rho \frac{\partial V^{\prime}}{\partial r}=-\frac{4 g \rho}{5 a} c^{\prime}\left(\mathrm{e}-e_{1}\right) r P_{2}, \\
\frac{\rho}{r} \frac{\partial V^{\prime}}{\partial \theta}=\frac{3 g \rho}{5 a} c^{\prime}\left(\mathrm{e}-e_{1}\right) r \sin 2 \theta,
\end{gathered}
$$

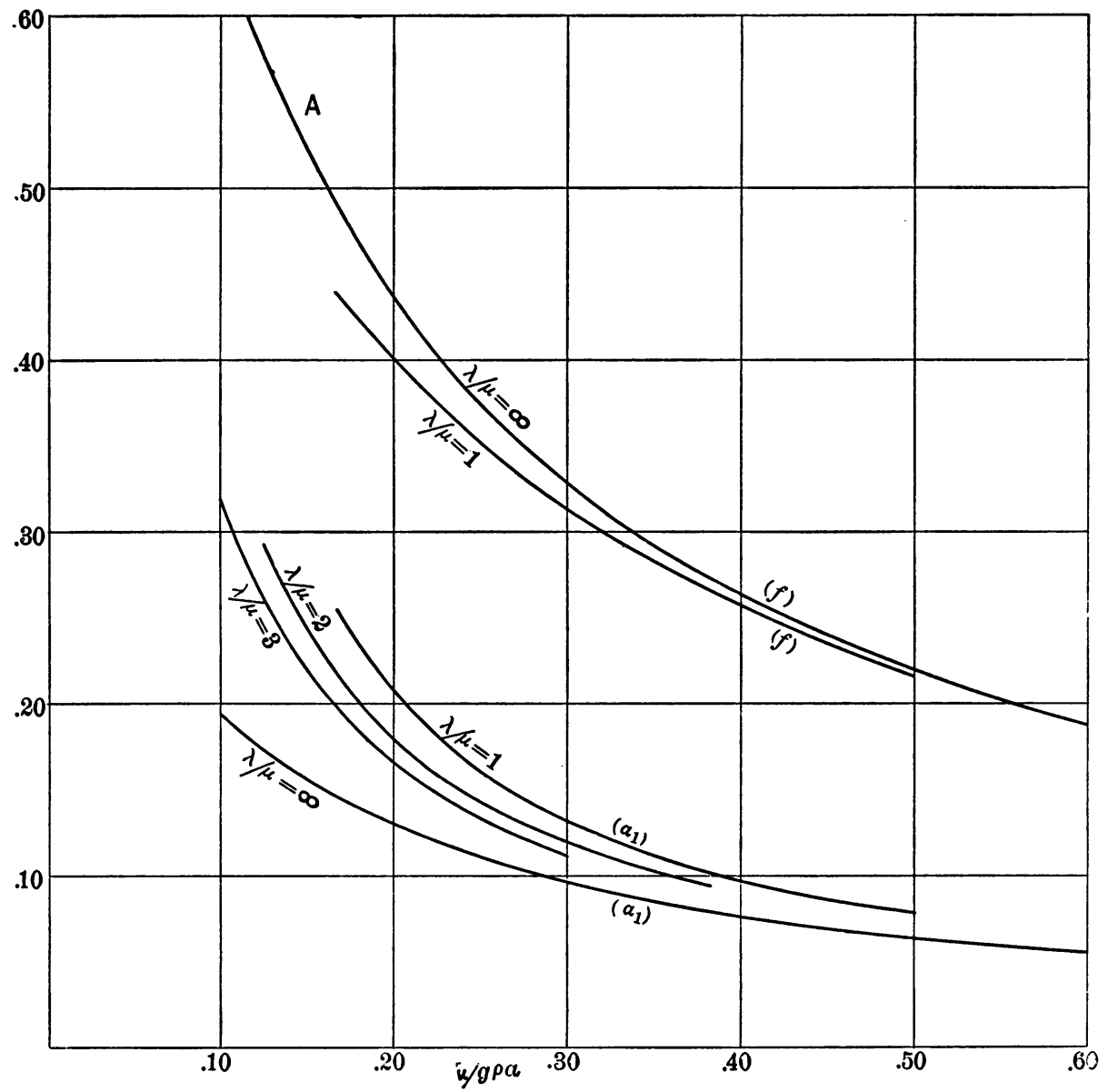

FIG. 3. Curves showing effect of compressibility upon angular displacement and external potential.

and therefore to (25) and (26) the terms

$$
\begin{array}{r}
-\frac{4}{5} g \rho a c^{\prime}\left(\mathrm{e}-e_{1}\right) x^{2}, \\
{ }_{5}^{3} g \rho a c^{\prime}\left(\mathrm{e}-e_{1}\right) x^{2} .
\end{array}
$$


(b) The weight of the ocean inequality per unit area is

$$
-\frac{2}{3} c^{\prime} \rho g a\left(\mathrm{e}-e_{1}\right) P_{2},
$$

hence there must be added to the second member of (32) the term

$$
\frac{2}{3} \rho g a c^{\prime}\left(\mathrm{e}-e_{1}\right) \text {. }
$$

§ 3. Value of ellipticity of ocean surface.

The value of e (the ellipticity of a surface which is level under the action of gravity and the disturbing forces) may be expressed in terms of $f, e_{1}$ and $c$, as follows :

For the solid sphere the value of $C-A$ is $I f$, while for the ocean inequality it is $I c^{\prime}\left(\mathrm{e}-e_{1}\right)$. Hence by a familiar result in the theory of potential,

$$
I\left[f+c^{\prime}\left(\mathrm{e}-e_{1}\right)\right]=\frac{2}{3} M a^{2}\left(\mathrm{e}-\frac{1}{2} c\right) .
$$

Or, since for a sphere of uniform density $I=\frac{2}{5} M a^{2}$, we have

$$
f+c^{\prime}\left(\mathrm{e}-e_{1}\right)=\frac{5}{3}\left(\mathrm{e}-\frac{1}{2} c\right) \text {, }
$$

whence

$$
\mathrm{e}=\frac{6 f-6 c^{\prime} e_{1}+5 c}{10-6 c^{\prime}}
$$

and

$$
\mathrm{e}-e_{1}=\frac{6 f-10 e_{1}+5 c}{10-6 c^{\prime}} .
$$

The terms to be added to (25), (26) and (32) thus become

$$
\begin{gathered}
\rho g a\left[-{ }_{5}^{4} c^{\prime} \frac{6 f-10 e_{1}+5 c}{10-6 c^{\prime}} x^{2}\right], \\
\rho g a\left[\frac{3}{5} c^{\prime} \frac{6 f-10 e_{1}+5 c}{10-6 c^{\prime}} x^{2}\right], \\
\rho g a\left[\frac{2}{3} c^{\prime} \frac{6 f-10 e_{1}+5 c}{10-6 c^{\prime}}\right] .
\end{gathered}
$$

\section{§4. Statement of solution.}

The values of $e$ and $\alpha$ for this case are expressed by the same equations $(A)$ and $(B)$ as before, but two of the three equations $(C),(D)$ and $(E)$ which determine the constants must be changed as follows.

In $(C)$ replace $\frac{10}{3} c$ by $\left[50 c+6 c^{\prime}\left(6 f^{\prime}-10 e_{1}\right)\right] /\left[3\left(5-3 c^{\prime}\right)\right]$.

Add to the first member of $(D)$ :

$$
\left(\frac{\lambda}{\mu}+2\right) b \frac{c^{\prime}\left(5 c+6 f-10 e_{1}\right)}{10-6 c^{\prime}} .
$$


Equation $(E)$ remains unchanged.

Equations $(C)$ and $(D)$ are thus replaced by the following:

$$
\begin{aligned}
4\left(A_{0}+A_{2}\right) & +\frac{140}{3 b(\lambda / \mu+2)} A_{2} \\
+ & \left(6 B_{2}-5 A_{2}\right)\left(2-\frac{20 \lambda / \mu}{3 b(\lambda / \mu+2)}-b N(b)\right) \\
& +\frac{12 c^{\prime}}{5-3 c^{\prime}} f-\frac{20 c^{\prime}}{5-3 c^{\prime}} e_{1}+\frac{50}{3\left(5-3 c^{\prime}\right)} c=0,
\end{aligned}
$$

$$
\left[2+\left(\frac{\lambda}{\mu}+2\right) b\right] A_{0}+\left[6+\left(\frac{\lambda}{\mu}+2\right) b\right] A_{2}-\left(6 B_{2}-5 A_{2}\right)
$$

$\left(D_{1}\right) \quad \times\left\{\frac{\lambda}{\mu}-\frac{b}{24}\left[\left(3 \frac{\lambda}{\mu}+2+\left(\frac{\lambda}{\mu}+2\right) b\right) L(b)+6 \frac{\lambda}{\mu} M(b)+2\left(\frac{\lambda}{\mu}+2\right) L^{\prime}(b)\right]\right\}$

$$
+\left(\frac{\lambda}{\mu}+2\right) b\left(\frac{3 c^{\prime}}{5-3 c^{\prime}} f-\frac{5 c^{\prime}}{5-3 c^{\prime}} e_{1}+\frac{5 c^{\prime}}{2\left(5-3 c^{\prime}\right)} c\right)=0 .
$$

When $f$ and $e_{1}$ are replaced by their values

$$
e_{1}=A_{0}+A_{2}+\frac{1}{24}\left(6 B_{2}-5 A_{2}\right) b L(b),
$$

(101) $f=A_{0}+A_{2}+\left(6 B_{2}-5 A_{2}\right)\left[\frac{1}{7}+\frac{b}{12}\left(2 G(b)-3 \frac{\lambda}{\mu} F(b)\right)\right]$,

the equations become

$$
\begin{gathered}
\frac{20\left(1-c^{\prime}\right)}{5-3 c^{\prime}}\left(A_{0}+A_{2}\right)+\frac{140}{3 b(\lambda / \mu+2)} A_{2}+\left(6 B_{2}-5 A_{2}\right) \\
\left(C^{\prime}\right) \quad \times\left[2-\frac{20 \lambda / \mu}{3 b(\lambda / \mu+2)}-b N(b)+\frac{4 c^{\prime}}{5-3 c^{\prime}}\left(\frac{3}{7}+\frac{1}{2} b G(b)-\frac{3 \lambda}{4 \mu} b F(b)-\frac{5}{24} b L(b)\right)\right] \\
\quad+\frac{50}{3\left(5-3 c^{\prime}\right)} c=0, \\
{\left[2+\left(\frac{\lambda}{\mu}+2\right) b \frac{5\left(1-c^{\prime}\right)}{5-3 c^{\prime}}\right]\left(A_{0}+A_{2}\right)+4 A_{2}-\left(6 B_{2}-5 A_{2}\right)} \\
\quad \times\left\{\frac{\lambda}{\mu}-\frac{1}{24}\left[\left(3 \frac{\lambda}{\mu}+2+\left(\frac{\lambda}{\mu}+2\right) b\right) b L(b)+6 \frac{\lambda}{\mu} b M(b)+2\left(\frac{\lambda}{\mu}+2\right) b L^{\prime}(b)\right]\right. \\
\left.\quad-\left(\frac{\lambda}{\mu}+2\right) b \frac{c^{\prime}}{5-3 c^{\prime}}\left[\frac{3}{7}+\frac{1}{2} b G(b)-\frac{3 \lambda}{4 \mu} b F(b)-\frac{5}{24} b L(b)\right]\right\} \\
\left(D^{\prime}\right) \quad+\left(\frac{\lambda}{\mu}+2\right) b \frac{5 c^{\prime}}{2\left(5-3 c^{\prime}\right)} c=0 .
\end{gathered}
$$


To these we add equation $(E)$ unchanged :

$\left(E^{\prime}\right) 3\left(A_{0}+A_{2}\right)+5 A_{2}+\left(6 B_{2}-5 A_{2}\right)\left[1+\frac{b}{8}\left(L(b)-2 M^{\prime}(b)\right)\right]=0$.

These three equations determine the constants $A_{0}, A_{2}, B_{2}$.

§ 5. Value of inertia-constant for combined sphere and ocean.

Let $f^{\prime}$ represent the value of $(C-A) / I$ for the whole system, then

which by $(96)$ reduces to

$$
f^{\prime}=f+c^{\prime}\left(e-e_{1}\right)
$$

$$
f^{\prime}=\frac{5}{5-3 c^{\prime}} f-\frac{5 c^{\prime}}{5-3 c^{\prime}} e_{1}+\frac{5 c^{\prime}}{2\left(5-3 c^{\prime}\right)} c .
$$

\$. Case of incompressibility.

If $\lambda=\infty$, equations $(A)$ and $(B)$ hold, and equations $\left(C^{\prime}\right)$ and $\left(D^{\prime}\right)$ require that

Also, on writing

$$
6 B_{2}-5 A_{2}=0 \text {. }
$$

$$
\frac{\lambda}{\mu}\left(6 B_{2}-5 A_{2}\right)=C,
$$

$\left(C^{\prime}\right),\left(D^{\prime}\right)$ and $\left(E^{\prime}\right)$ become

$$
\begin{gathered}
C-7 A_{2}-\frac{3\left(1-c^{\prime}\right)}{5-3 c^{\prime}} b^{\prime}\left(A_{0}+A_{2}\right)-\frac{5 b^{\prime}}{2\left(5-3 c^{\prime}\right)} c=0 \\
C-4 A_{2}-\left(2+\frac{5\left(1-c^{\prime}\right)}{5-3 c^{\prime}} b^{\prime}\right)\left(A_{0}+A_{2}\right)-\frac{5 c^{\prime} b^{\prime}}{2\left(5-3 c^{\prime}\right)} c=0 \\
3\left(A_{0}+A_{2}\right)+5 A_{2}=0
\end{gathered}
$$

The solution of these equations gives

$$
\begin{aligned}
& A_{0}=\frac{20\left(1-c^{\prime}\right) b^{\prime},}{19\left(5-3 c^{\prime}\right)+10\left(1-c^{\prime}\right) b^{\prime}} c, \\
& A_{2}=-\frac{15\left(1-c^{\prime}\right) b^{\prime}}{38\left(5-3 c^{\prime}\right)+20\left(1-c^{\prime}\right) b^{\prime}} c, \\
& B_{2}=-\frac{25\left(1-c^{\prime}\right) b^{\prime}}{76\left(5-3 c^{\prime}\right)+40\left(1-c^{\prime}\right) b^{\prime}} c,
\end{aligned}
$$

If $c^{\prime}=0$, these reduce to (85), as of course they should. 


\section{§ 7. Numerical results.}

For the purpose of showing the order of importance of the ocean effect, computations have been made with a value of $c^{\prime}$ equal to the ratio of the density of sea-water to the mean density of the earth. This value is taken as 5/27.

The computations made cover the two cases $\lambda / \mu=1, \lambda / \mu=\infty$, and the results are shown in the accompanying table and diagrams.

For the case of incompressibility

$$
A_{0}=\frac{22 b^{\prime}}{114+11 b^{\prime}} c=\frac{22}{114 \frac{\mu}{g \rho a}+11} c, \quad A_{2}=-\frac{3}{8} A_{0}, \quad B_{2}=-\frac{5}{16} A_{0}
$$

$$
\begin{gathered}
\frac{e}{c}=\frac{22}{114 \frac{\mu}{g \rho a}+11}\left(1-\frac{3}{8} x^{2}\right), \\
\frac{\alpha}{c}=\frac{11}{114 \frac{\mu}{g \rho a}+11}\left(1-\frac{5}{8} x^{2}\right), \\
\frac{e_{1}}{c}=\frac{f}{c}=\frac{1}{c}\left(A_{0}+A_{2}\right)=\frac{5}{\frac{456}{11} \frac{\mu}{g \rho a}+4} .
\end{gathered}
$$

The last expression is written in a form for comparison with the value without ocean effect:

From (102),

$$
\frac{e_{1}}{c}=\frac{f}{c}=\frac{5}{38 \frac{\mu}{g \rho a}+4}
$$

Values of $e_{1}, f$ and $f^{\prime}$ for this case, also corresponding values without ocean effect, are given in Table IV, while the same results are shown graphically in Fig. 4.

For $\lambda=\mu$, computations have been made by means of equations $\left(A^{\prime}\right),\left(B^{\prime}\right)$, $\left(C^{\prime}\right),\left(D^{\prime}\right),\left(E^{\prime}\right)$, with the results given in Table V and shown graphically in Fig. 5.

Inspection of the curves shows that the effect of the ocean on the strain of the sphere is quite appreciable even in the case of incompressibility, and is very considerable in the case $\lambda=\mu$. A fact of equal importance in the interpretation of observational results as to the yielding of the earth is shown by the curves representing $f^{\prime}$. These are nearly identical for the two cases $\lambda=\infty$, $\lambda=\mu$ (Figs. 4 and 5), and in both cases are very considerably above the curves representing $f$. It thus appears that an important part of the change of external potential is due to the ocean $-a$ result which will be referred to again in the following discussion of the yielding of the earth. 


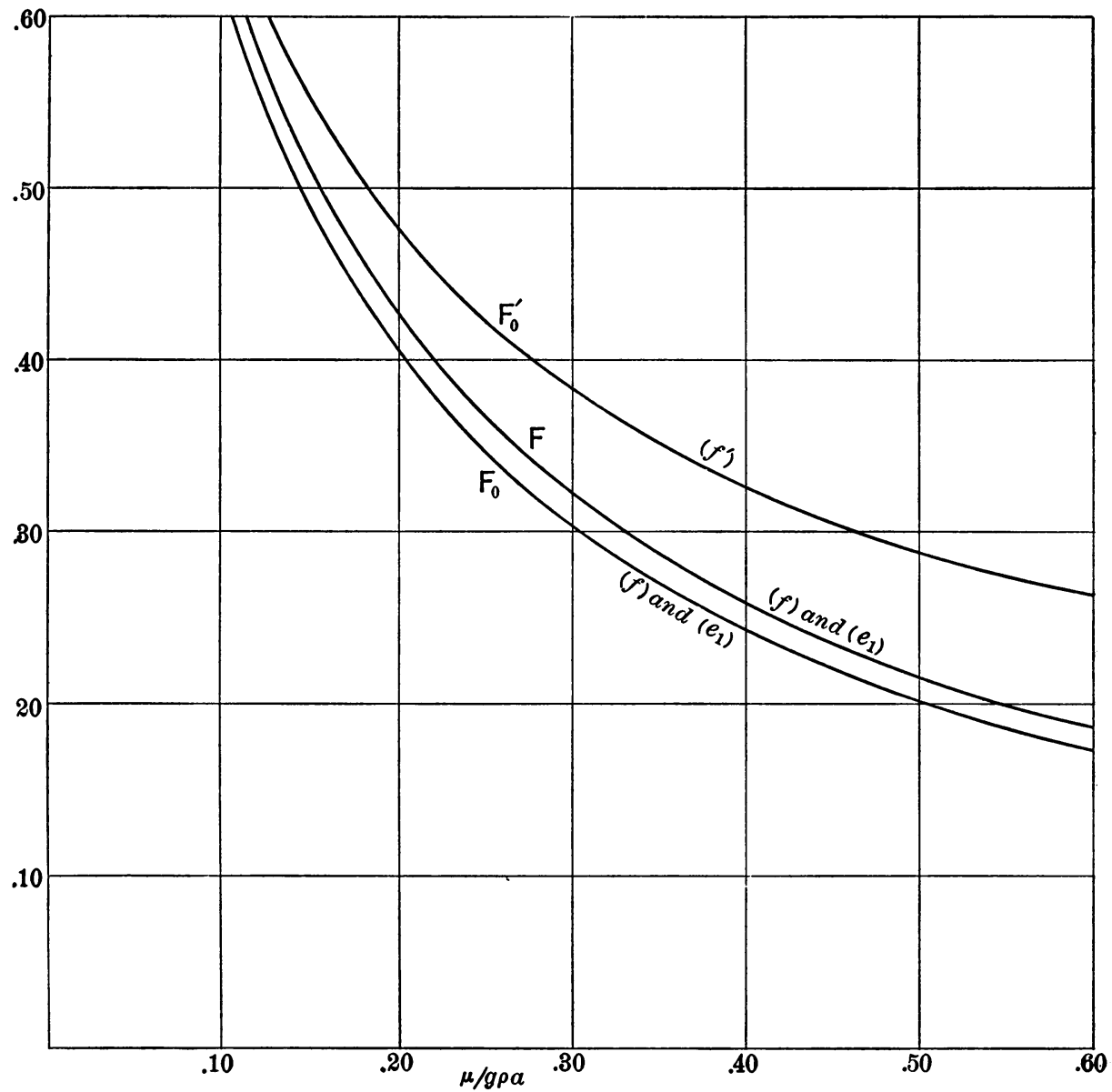

FIG. 4. Curves showing effect of ocean in case $\lambda=\infty$. ( $F_{0}$ and $F_{0}^{\prime}$ with ocean, $F$ without.) TABLE IV. Effect of ocean in case $\lambda=\infty$.

\begin{tabular}{l|c|c|c|c|c}
\hline \hline \multirow{2}{*}{$\mu$} & \multicolumn{2}{|c|}{ Without ocean. } & \multicolumn{2}{c}{ With ocean. } \\
\cline { 2 - 6 } & $\frac{a_{1}}{c}$ & $\frac{e_{1}}{c}=\frac{f}{c}$ & $\frac{a_{1}}{c}$ & $\frac{e_{1}}{c}=\frac{f}{c}$ & $\frac{f^{\prime}}{c}$ \\
\hline 0 & .375 & 1.250 & .375 & 1.250 & 1.250 \\
.05 & .254 & .848 & .247 & .823 & .858 \\
.10 & .193 & .642 & .184 & .613 & .666 \\
.15 & .155 & .516 & .146 & .488 & .551 \\
.20 & .129 & .431 & .122 & .407 & .477 \\
.25 & .111 & .370 & .104 & .348 & .423 \\
.30 & .097 & .325 & .091 & .304 & .383 \\
.35 & .087 & .289 & .081 & .270 & .352 \\
.40 & .078 & .260 & .073 & .243 & .327 \\
.45 & .071 & .237 & .066 & .221 & .307 \\
.50 & .065 & .217 & .061 & .202 & .289 \\
.55 & .060 & .201 & .056 & .187 & .275 \\
.60 & .056 & .187 & .052 & .173 & .263 \\
\hline
\end{tabular}




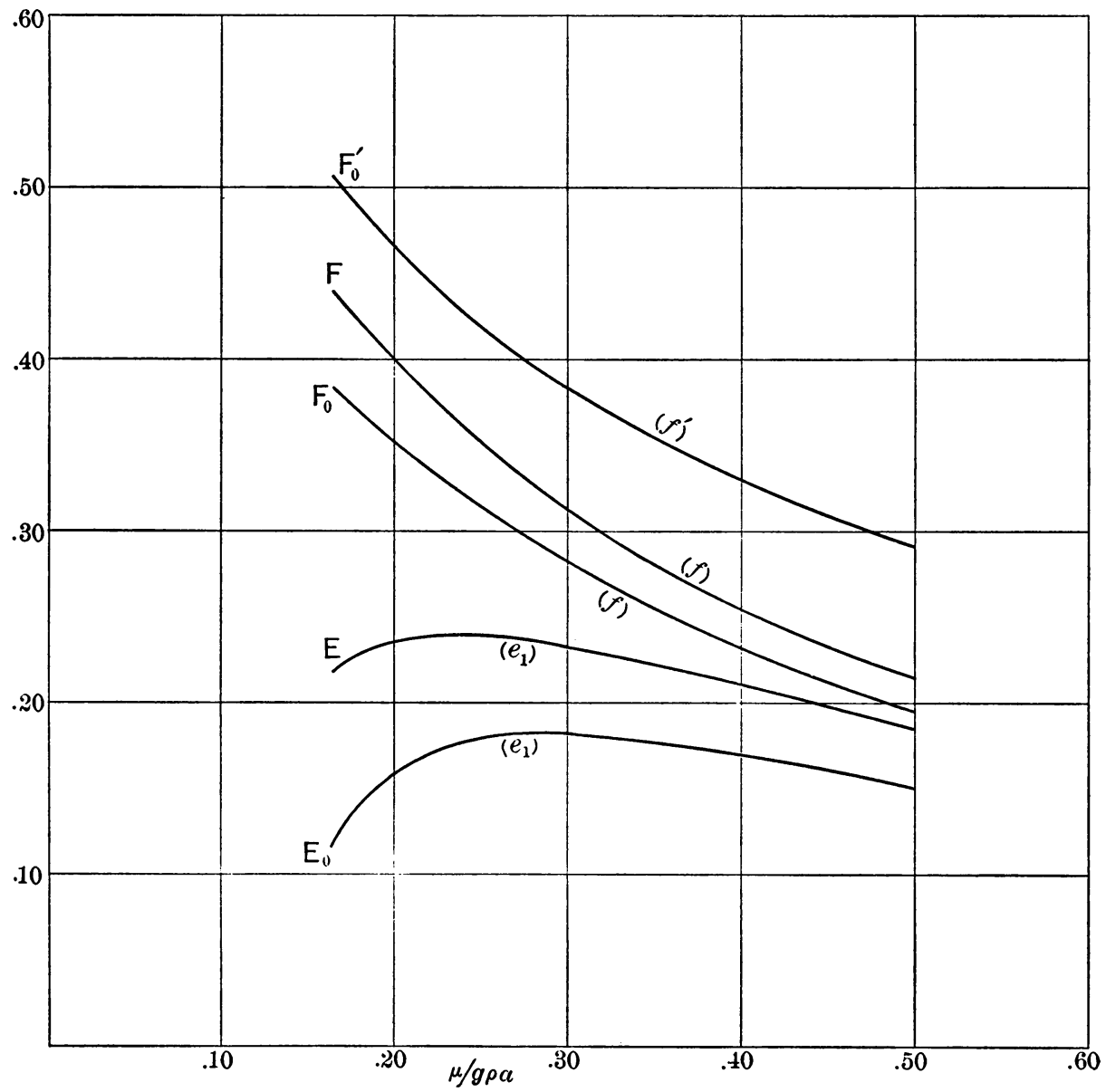

FIG. 5. Curves showing effect of ocean in case $\lambda=\mu$. (Curves $E, F$ without ocean; $E_{0}, F_{0}$, $F_{0}^{\prime}$ with ocean.)

TABLE V. Effect of ocean in case $\lambda=\mu$.

\begin{tabular}{|c|c|c|c|c|c|c|c|c|}
\hline \multirow{2}{*}{$b$} & \multirow{2}{*}{$\frac{\mu}{g \rho i}$} & \multicolumn{3}{|c|}{ Without ocean. } & \multicolumn{4}{|c|}{ With ocean. } \\
\hline & & $\frac{a_{1}}{c}$ & $\frac{e_{1}}{c}$ & $\frac{f}{c}$ & $\frac{a_{1}}{c}$ & $\frac{e_{1}}{c}$ & $\frac{f}{c}$ & $\frac{f^{\prime}}{c}$ \\
\hline 2 & .167 & .253 & .216 & .436 & .257 & .119 & .379 & .505 \\
\hline 1.8 & .185 & .224 & .230 & .415 & .229 & .145 & .365 & .485 \\
\hline 1.6 & 208 & .196 & .238 & .390 & .198 & .163 & .345 & .458 \\
\hline 1.4 & .238 & .169 & .241 & .361 & .170 & .176 & .322 & .429 \\
\hline 1.2 & .278 & .142 & .237 & .327 & .143 & .181 & .294 & .397 \\
\hline 1.0 & .333 & .117 & .225 & .289 & .118 & .179 & .263 & .363 \\
\hline .8 & .417 & .093 & .204 & .246 & .094 & .167 & .225 & .322 \\
\hline .667 & .500 & .077 & .184 & .213 & .077 & .151 & .194 & 291 \\
\hline
\end{tabular}




\section{EFFeCT OF COMPRESSIBILITY UPON ESTIMATES OF THE RIGIDITY OF THE EARTH.}

The estimates of the rigidity of the earth, made originally by LORD KELviN and since by others, are based upon a comparison of what is known regarding the actual yielding of the earth under known disturbing forces with the computed yielding of an ideal body under like forces. This ideal body is a homogeneous, elastic, incompressible sphere of mass and size equal to those of the earth. It is, of course, not to be expected that such a comparison can lead to anything more than a rough average value of the modulus of rigidity of the material of the earth, since the actual earth is certainly far from uniform in density, and probably far from uniform in elastic properties. It is, however, a matter of some interest to notice in what way these estimates are modified when compressibility is taken into account. In answering this question it is needful to refer briefly to the present status of the evidence regarding the actual yielding of the earth.*

\section{§1. Nature of evidence of yielding of the earth.}

Evidence regarding the actual yielding of the earth relates to the two quantities above represented by $e_{1}$ and $f^{\circ}$. The former is the surface ellipticity due to strain, the latter measures the change in the gravitational potential at the earth's surface due to the strain. The number $f$ is, in fact, the value of $(C-A) / I$ due to the strain. It may be remarked that for a homogeneous incompressible body $e_{1}$ and $f$ are equal, but for a compressible body $f$ depends upon the values of both $e$ and $\alpha$ throughout the body.

There are three lines of evidence of yielding of the earth to tidal or centrifugal forces: $(a)$ Tidal observations, (b) experiments with the horizontal pendulum, $(c)$ the movement of the axis of rotation within the earth as determined by latitude observations.

(a) Nature of evidence from tidal observations. The height of an equilibrium-tide at any locality can be computed on the assumption that the solid earth is unyielding, also on the assumption that $f$ and $e_{1}$ have any definite values. A comparison of these results with the observed tidal oscillation gives evidence as to the actual values of $f$ and $e_{1}$. It may, in fact, be shown without difficulty that if the attraction of the ocean itself be neglected, the ratio of the actual fluctuation of the water relative to the solid ground to the fluctuation on the assumption of no yielding of the solid earth is

* A clear and non-technical summary of this evidence is given by G. H. DARwin in a paper entitled "The Rigidity of the Earth," in Rivista di Scienza, vol. 5 (1909). See also a paper by A. E. H. Love entitled "The Yielding of the Earth to Disturbing Forces," in the Proceedings of the Royal Society, Series A, Vol. 84. 


$$
\frac{c+f-2 e_{1}}{c}=1+\frac{f}{c}-2 \frac{e_{1}}{c} .
$$

It is here assumed that the tide has its equilibrium value, an assumption which can reasonably be made only in the case of tides of long period. It is therefore only the fortnightly and monthly tides that have been available for this comparison.

It should be said also that for full generality the term $f / c$ must be multiplied by $3 I / M a^{2}$. For a homogeneous sphere the value of this is $6 / 5$, but for the earth it is very nearly 1 . It is the actual value for the earth that must be used in the application made below in $\$ 2$.

(b) Nature of the evidence from experiments with the horizontal pendulum. If the change in the direction of apparent gravity relative to the earth at any locality due to tidal forces can be measured, a comparison of this effect with the effect computed on the assumption of absolute rigidity of the earth will give evidence as to the actual yielding of the earth. The actual effect is so minute that it must be greatly magnified in order to become measurable. This magnification has been accomplished by means of the horizontal pendulum. It is not difficult to show that the actual deflection of such a pendulum due to tidal forces bears the same ratio to the deflection that would oceur if the earth did not yield as the amplitude of an equilibrium tide bears to the ampliture it would have if the earth were unyielding. The quantity determined from horizontal pendulum observations is therefore identical with that inferred from tidal observations.

(c) Nature of the evidence from the periodical variation of latitudes. The periodic motion of the axis of rotation within the earth furnishes evidence as to the yielding of the earth to centrifugal forces. This evidence is of a different character from that obtained from the tides or the horizontal pendulum, in that it leads to an estimate of the value of $f / c$ instead of to a relation between $f / c$ and $e_{1} / c$. The change in the value of $(C-A) / I$ due to the yielding of the earth to given centrifugal forces may be inferred directly from a comparison of the actual period of the free nutation of the earth's axis with the period computed for an unyielding body having the earth's actual figure. It may, in fact, be shown that the actual nutation period is to a close approximation the same as that of an unyielding body having the figure which the earth would assume if centrifugal forces were annulled.*

* This was shown by S. S. Hougr (Philosophical Transactions of the Royal Society, ser. $A, 1896)$ for the case of a homogeneous, incompressible elastic spheroid, and has been proved by J. I.ARMOR and others to hold without other restriction as to the character of the body than that its density and elastic properties have approximate spherical symmetry. A proof of this was given by the present writer in a paper presented to the American Irathematical Society, Sau Francisco Section, Dec. 20, 1902, of which only an abstract was published, Bulletin of this Scciety, Vol. 9 (1903), p. 299. The reasoning employed was identical with that published by Larior, Proceedings of the Royal Society, ser. $A$, vol. 82 (1909). 
\$2. Numerical results expressing actucl yielding of the earth.

The actual value of $(C-A) / I$ for the earth is $1 / 306$, so that the period of free nutation if it were absolutely rigid would be 306 sidereal days. The period of the movement of the axis about its mean position, as inferred from the observations of the International Latitude Service carried on systematically since 1899, has varied from 427 days to 442 days. The former gives

the latter,

$$
f=\frac{1}{30}-\frac{1}{42} \pi=.00093 \text {; }
$$

$$
f=\frac{1}{306}-\frac{1}{42}=.00100 .
$$

Since the ratio of equatorial centrifugal force to gravity is $c=1 / 289$, the corresponding values of $f / c$ are .269 and .289 . The former of these values has hitherto usually been accepted. It is nearly equal to $4 / 15$.

The first analysis of tidal observations, with reference to the question of the yielding of the earth, was made by G. H. DARwiN, the data and results being given in Thomson and Tait's Natural Philosophy. From all the data then available which were regarded as trustworthy, the value found for the ratio of the actual tidal amplitude to that computed on the assumption of an absolutely rigid earth was $.676 \pm .076$; but a separate analysis of the more consistent observations gave $.931 \pm .056$. The result was not regarded by the author as conclusive.* Analyses since made by others have been confirmatory of the former of these values, and about the same result is inferred from observations with the horizontal pendulum. $\dagger$

If this result be accepted, we have (very nearly)

$$
1+{ }_{c}^{f}-2{ }_{c}^{e_{1}}=\frac{2}{3} .
$$

And if in this we substitute

there results

$$
\frac{f}{c}=\begin{gathered}
4 \\
15
\end{gathered}
$$

$$
\frac{e_{1}}{c}=\frac{3}{10} \text {. }
$$

§3. Comparison of actual yielding with that of an ideal elastic solid.

It is pointed out by LOVE $\ddagger$ that the above values of $f / c$ and $e_{1} / c$ cannot coexist in the case of a homogeneous, incompressible elastic solid having the earth's size and mass, and it is easily seen from the results found in the first

* "We thus see that the more consistent observations seem to bring out the tides more nearly to their theoretical equilibrium values with no elastic yielding of the solid." G. H. DARWIN, in Thomson and Tait's Natural Philosophy, Part II, p. 460 (edition of 1890).

† W. Schweydar, Beiträge zur Geophysik, vol. 9 (1908); O. Hecker, Veröffentlichung des $\mathrm{Kgl}$. Preussischen Geodätischen Institutes, No. 32, Berlin (1907).

$\ddagger$ Loc. cit. 
part of this paper that the discrepancy is increased rather than diminished if the effect of compressibility is considered. This is shown clearly by Fig. 6 , in which the curves represent the relation between $e_{1} / c$ and $f / c$ for several values of $\lambda / \mu$, while the straight line $A B$ represents equation (115), or

$$
2 \frac{e_{1}}{c}-\frac{f}{c}=\frac{1}{3} \text {. }
$$

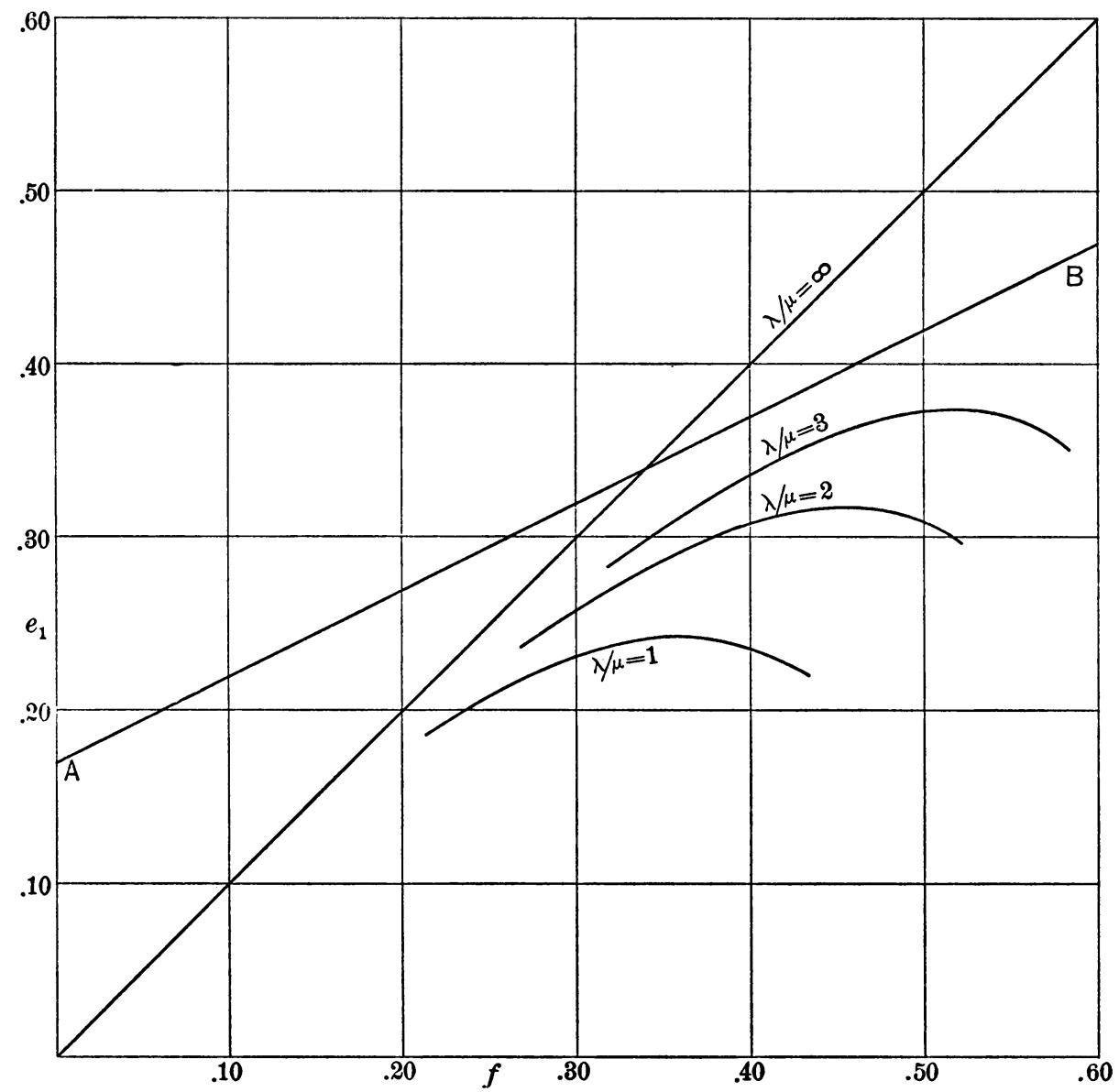

Fia. 6. Curves showing relation between $e_{1}$ and $f$ for several values of $\lambda / \mu$.

As $\lambda / \mu$ increases, the curves approach the limiting line $e_{1}=f$; and until $\lambda / \mu$ reaches a value considerably greater than 3 , the curves will fail to intersect $A B$. It is obvious that if $\lambda / \mu$ be taken great enough so that (116) can be satisfied the value of $f / c$ will be greater than $\frac{4}{15}$; being in fact $\frac{1}{3}$ for $\lambda=\infty$, and greater than $\frac{1}{3}$ for any finite value of $\lambda / \mu$.

It may be remarked that the discrepancy between 
and

$$
\frac{f}{\mathrm{c}}=\frac{1}{3}, \quad \frac{e_{1}}{c}=\frac{1}{3},
$$

$$
\stackrel{f}{c}=\frac{4}{15}, \quad \stackrel{e_{1}}{c}=\frac{3}{10},
$$

is after all not remarkably great, considering the great delicacy of the observations upon which equation (116) is based. It may perhaps be still open to question whether the accepted values are so thoroughly reliable as to exclude the possibility of errors of the magnitude of these discrepancies.*

It may be noted, also, that a value of $1+f / c-2 e_{1} / c$ much less than the greater of Darwin's results would remove the discrepancy. Thus the following

\begin{tabular}{|c|c|c|c|}
\hline$\frac{\lambda}{\mu}$ & $\stackrel{i}{i}$ & $\begin{array}{l}e_{1} \\
i\end{array}$ & $1+\frac{f}{c}-2 \frac{e_{1}}{c}$ \\
\hline$\infty$ & .270 & .270 & .730 \\
\hline 3 & .270 & .245 & .780 \\
\hline 2 & .270 & .235 & .800 \\
\hline 1 & .270 & $.2: 0$ & .830 \\
\hline
\end{tabular}
values would be consistent for the ideal homogeneous sphere :

Although the value of the surface ellipticity accepted as most reliable is more nearly consistent with the computed value for an incompressible than for a compressilble sphere, it can hardly be supposed that this furnishes any important evidence as to the actual compressibility of the materials composing the earth. As stated above, only a roughly approximate agreement is to be expected between the behavior of the actual earth and that of an ideal body assumed to be uniform in density and elastic properties. Moreover, there are certain questions not generally considered wnen appear to require attention in any complete study of the theoretical basis of the comparison. One of these questions relates to the effect of the ocean.

* The most elaborate and apparently the most trustworthy series of observations heretofore made with the horizontal pendulum are those of HECKER (loc. cit.). A remarkable feature of the results obtained for the lunar effect upon the yielding of the earth is the variation of this effect with the azimuth of the moon. The value found for the ratio of the actual deflection of gravity to the deflection computed for a rigid earth is a minimum when the moon is on the meridian, and increases continually with the azimuth. The minimum value of the ratio is about 0.36 , for azimuth $45^{\circ}$ it is about 0.50 , and for azimuth $67^{\circ}$ about 0.90 ; and although as the azimuth approaches $90^{\circ}$ the ratio becomes indeterminate, it appears to approach unity. No satisfactory explanation of this variation seems to have been suggested. If it is accepted as proving that the yielding of the earth varies greatly with the azimuth of the disturbing body, it appears to render of little value conclusions based upon the ordinary theory which assumes that the yielding is symmetrical ahout the axis of symmetry of the disturbing forces. At all events these results can hardly be regarded as establishing any definite value of the ratio of yielding. [The above numerical values of the ratio for different azimuths were ohtained by measurement from the diagram given by HEckek showing the observed lunar effect and the effect computed for a rigid earth; assuming that the deflection of apparent gravity al ways agrees in azimuth with the moon.] HEcker's results are discussed by G. H DARWIN in the paper already cited. This paper was received too late to permit here any 'quotation of suggestive comments. 
§4. Effect of the ocean on estimates of the yielding of the earth.

The question as to the effect of the ocean involves two parts, - the first, what effect has the ocean on the actual yielding of the solid earth, and the second, what direct effect has the ocean on the observational results?

While no exact answer can be given to either of these questions, something can be inferred as to the degree of importance of the ocean effect from the results reached in Part II of this paper, and shown in Tables IV and V, and in Figs. 4 and 5. These results apply to the two cases $\lambda=\infty$ and $\lambda / \mu=1$. It appears that the effect of the ocean upon the strain of the sphere is quite appreciable in both cases, and in the case $\lambda=\mu$ becomes of considerable importance. Although the angular displacement $\alpha_{1}$ is affected but slightly, both $e_{1}$ and $f$ are very considerably changed by the influence of the ocean.

Moreover, while these quantities are both materially decreased, the total external potential due to the solid sphere and ocean is considerably greater than the potential when the ocean is absent. This is true both for $\lambda=\mu$ and for $\lambda=\infty$, as is seen from the values of $f^{\prime} / c$. (It is of interest to note that the curves showing the relation between $f^{\prime} / c$ and $\mu / g \rho a$ are nearly identical for the two cases.)

In estimating the value of $\mu$ from the value of $(C-A) / I$ inferred from the prolongation of the period of free nutation, it is therefore important to know whether the quantity determined applies to the solid earth alone or to the combined solid earth and ocean. Thus if it applies to the solid earth alone, the value $f / c=0.27$ would correspond to

$$
\frac{\mu}{g \rho a}=\text { about } 0.37
$$

while if 0.27 is the value of $f^{\prime} / c$, we find

$$
\stackrel{\mu}{g \rho a}=\text { about } 0.57 \text {. }
$$

The former would give $\mu=1.30 \times 10^{12}$, while the latter would give $\mu=2 \times 10^{12}$. These values are in C. G. S. units (dynes per square centimeter), the value of $g \rho a$ in these units being taken as $3.5 \times 10^{12}$.

Whether the change in the value of $(C-A) / I$ due to centrifugal forces, as inferred from the nutation period, applies to the solid earth alone, or whether an important part of it is due to the change in configuration of the ocean, is a question which must be answered in the light of the theory of the effect of yielding upon the nutation period. It seems a reasonable assumption that, for a disturbance for so long a period as 427 days, the ocean always has its equilibrium figure. If this be assumed in applying the equation of angular momen- 
tum,* it is difficult to avoid the conclusion that the change in the value of $(C-A) / I$ inferred from the prolongation of the nutation period includes the yielding of the ocean; in other words, that the number $4 / 15$ is the value of $f^{\prime} / c$ rather than of $f / c$.

What effect the ocean may have upon observations with the horizontal pendulum is a more difficult question, because the equilibrium theory must be discarded in dealing with tides of short period.

\section{Strain of a COMpressible, gravitating elastic sphere due to DISTURBING FORCES OF WHICH THE POTENTIAL IS A SPHERICAL HARMONIC OF ANY DEGREE NOT LESS THAN THE SECOND.}

The following solution of this problem follows the general lines of the solution already given for the case in which the degree of the harmonic is 2 . It in fact includes that as a special case. In comparing the algebraic results it must be noted that there is a slight change in the notation; the quantities $e$ and $\alpha$ as used below must be multiplied by the factor $-\frac{3}{2}$ in order to agree with $e$ and $\alpha$ in the solution given above.

\section{§1. Physical analysis of the problem.}

The physical analysis is identical with that already given in the special case, and need not be repeated.

§ 2. Formation of the equations of equilibrium of an element of volume.

(a) General form of equations in polar coördinates. The polar coördinates being $r, \theta, \phi$, let the corresponding displacements be

$$
u_{r}, u_{\theta}, u_{\phi},
$$

the cubical expansion $\Delta$, and the three components of rotation $\varpi_{r}, \varpi_{\theta}, \varpi_{\phi}$. Also let the components of total bodily force (per unit volume) be $\rho F_{r}, \rho F_{\theta}, \rho F_{\phi}$. Then the equations of equilibrium for a volume element are the following: $\dagger$

$$
\begin{aligned}
& (\lambda+2 \mu) \frac{\partial \Delta}{\partial r}-\frac{2 \mu}{r \sin \theta}\left[\frac{\partial}{\partial \theta}\left(\varpi_{\phi} \sin \theta\right)-\frac{\partial \varpi_{\theta}}{\partial \phi}\right]=-\rho F_{r}, \\
& (\lambda+2 \mu) \frac{1}{r} \frac{\partial \Delta}{\partial \theta}-\frac{2 \mu}{r \sin \theta}\left[\frac{\partial \varpi_{r}}{\partial \phi}-\frac{\partial}{\partial r}\left(r \varpi_{\phi} \sin \theta\right)\right]=-\rho F_{\theta}, \\
& (\lambda+2 \mu) \frac{1}{r \sin \theta} \frac{\partial \Delta}{\partial \phi}-\frac{2 \mu}{r}\left[\begin{array}{c}
\partial \\
\partial r
\end{array}\left(r \varpi_{\theta}\right)-\frac{\partial \varpi_{r}}{\partial \theta}\right]=-\rho F_{\phi} ;
\end{aligned}
$$

* In the manner employed by LARMOR in the paper cited above.

† See Love, Treatise on the Mathematical Theory of Elasticity, 2d edition (1906), p. 138, for the first members of these equations; p. 56 , for the values of $\Delta, \varpi_{r}, \varpi_{\theta}, \varpi_{\phi}$. 
while the cubical expansion and the components of rotation are given by the following equations :

$$
\begin{gathered}
\Delta=\frac{1}{r^{2} \sin \theta}\left[\frac{\partial}{\partial r}\left(r^{2} u_{r} \sin \theta\right)+\frac{\partial}{\partial \theta}\left(r u_{\theta} \sin \theta\right)+\frac{\partial}{\partial \bar{\phi}}\left(r u_{\phi}\right)\right], \\
2 \varpi_{r}=\frac{1}{r^{2} \sin \theta}\left[\frac{\partial}{\partial \theta}\left(r u_{\phi} \sin \theta\right)-\frac{\partial}{\partial \phi}\left(r u_{\theta}\right)\right], \\
2 \varpi_{\theta}=\frac{1}{r \sin \theta}\left[\frac{\partial u_{r}}{\partial \phi}-\frac{\partial}{\partial r}\left(r u_{\phi} \sin \theta\right)\right], \\
2 \varpi_{\phi}=\frac{1}{r}\left[\frac{\partial}{\partial r}\left(r u_{\theta}\right)-\frac{\partial u_{r}}{\partial \theta}\right] .
\end{gathered}
$$

(b) Assumptions. The potential of the disturbing forces, being a spherical solid harmonic of degree $i$, is proportional to

$$
S_{i} r^{i}
$$

in which $S_{i}$ is a spherical surface harmonic, and therefore satisfies the partial differential equation

$$
\begin{array}{ccc}
1 & \partial^{2} S_{i} \\
\sin ^{2} \theta & \begin{array}{c}
1 \\
\partial \phi^{2}
\end{array} & \begin{array}{c}
\partial \\
\sin \theta
\end{array} \partial \theta
\end{array}\left(\sin \theta \frac{\partial S_{i}}{\partial \theta}\right)+i(i+1) S_{i}=0 .
$$

It will be assumed that

$$
\begin{aligned}
& u_{r}=u S_{i}, \\
& u_{\theta}=v \begin{array}{c}
\partial S_{i} \\
\partial \theta
\end{array} \\
& u_{\phi}=v \frac{1}{\sin \theta} \partial S_{i},
\end{aligned}
$$

in which $u$ and $v$ are independent of $\theta$ and $\phi$. These assumptions give by virtue of $(124)$

$$
\begin{aligned}
& \Delta=\left[\begin{array}{cc}
\frac{1}{r^{2}} d\left(r^{2} \prime\right) & d r
\end{array}\right. \\
& 2 w_{r}=0 \text {, } \\
& 2 \omega_{\theta}=\frac{1}{r}\left[u-\begin{array}{c}
l(r v) \\
d r
\end{array}\right] \sin \theta \quad \partial \phi, \\
& 2 \sigma_{\phi}=-\frac{1}{r}\left[u-\frac{d(r v)}{d r}\right] \frac{\partial S_{i}}{\partial \theta^{-}},
\end{aligned}
$$


and reduce (117), (118) and (119) to the following:

$\left[(\lambda+2 \mu) \frac{d}{d r}\left(\frac{1 d\left(r^{2} u\right)}{r^{2}-d r^{-}}-i(i+1) \frac{v}{r}\right)+i(i+1) \frac{\mu}{r^{2}}\left(\frac{d(r v)}{d r}-u\right)\right] S_{i}=-\rho F_{r}$

$$
\left[(\lambda+2 \mu) \frac{1}{r}\left(\frac{1}{r^{2}} \frac{d\left(r^{2} u\right)}{d r}-i(i+1)_{r}^{v}\right)+\frac{\mu d}{r} \frac{d}{d r}\left(\frac{d(r v)}{d r}-u\right)\right] \frac{\partial S_{i}}{\partial \theta}=-\rho F_{\theta},
$$

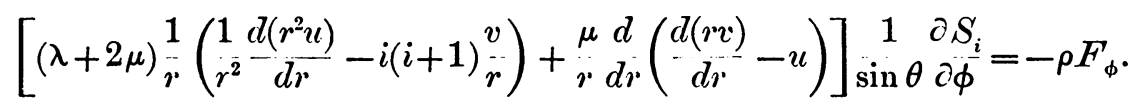

(c) Bodily forces. We now proceed to express the values of (1) the disturbing forces, (2) the gravitational force due to the change of density of the attracted element, (3) the attraction due to the change of configuration of the attracting mass.

(1) Disturbing forces. Let the potential of the disturbing forces be

$$
W_{i}=-\frac{2}{3 i} \operatorname{cga} a_{a^{i}}^{r^{2}} S_{i}
$$

then the terms in $\rho F_{r}, \rho F_{\theta}, \rho F_{\phi}$ representing these forces are

$$
\begin{gathered}
\rho \frac{\partial W_{i}}{\partial r}=-\frac{2}{3} \operatorname{cog} a \frac{r^{i-1}}{a^{i}} S_{i}, \\
\frac{\rho \partial W_{i}}{r}=-\frac{2}{\partial i} \operatorname{cog} a \frac{r^{i-1} \partial S_{i}}{a^{i}} \partial \theta^{-}, \\
\frac{\rho}{r \sin \theta} \frac{\partial W_{i}}{\partial \phi}=-\frac{2}{3 i} \operatorname{coga} \frac{r^{i-1}}{a^{i}} \sin \theta \partial S_{i}
\end{gathered}
$$

(2) Attraction of original mass-configuration upon increment of mass of element. The attraction of the original mass-configuration per unit mass upon an element distant $r$ from the center is

$$
-g \stackrel{r}{a}
$$

The increment of density is

$$
-\rho \Delta=-\rho\left[\begin{array}{c}
1 d\left(r^{2} u\right) \\
r^{2} d r
\end{array}-i(i+1)_{r}^{v} \cdot S_{i} .\right.
$$

Hence the attraction per unit volume, due to the increment of density of the attracted elemeni, is

$$
\begin{aligned}
\left(-g \frac{r}{a}\right)(-\rho \Delta)=g \rho \frac{r}{a}\left[\frac{1}{r^{2}}\right. & \left.\frac{d\left(r^{2} u\right)}{d r}-i(i+1) \frac{v}{r}\right] S_{i} \\
& =g \rho a \frac{r}{a^{2}}\left[\frac{d u}{d r}+2 \frac{u}{r}-i(i+1) \frac{v}{r}\right] S_{i} .
\end{aligned}
$$


(3) Attraction due to increment of density of attracting mass. The potential at a point $(r, \theta, \phi)$ due to a shell of density $D^{\prime} S_{i}$, radius $r^{\prime}$ and thickness $d r^{\prime}$ is

$$
\begin{array}{ll}
\frac{4 \pi \gamma}{2 i+1} \frac{r^{i+2}}{r^{i+1}} D^{\prime} S_{i} d r^{\prime} & \left(r>r^{\prime}\right), \\
\frac{4 \pi \gamma}{2 i+1} \frac{r^{i}}{r^{i-1}} D^{\prime} S_{i} d r^{\prime} & \left(r<r^{\prime}\right) .
\end{array}
$$

On replacing $D^{\prime} S_{i}$ by

$$
-\rho \Delta^{\prime}=\rho\left[i(i+1) \frac{v^{\prime}}{r^{\prime}}-\frac{1}{r^{\prime 2}} \frac{d\left(r^{\prime 2} u^{\prime}\right)}{d r^{\prime}}\right] S_{i},
$$

the potential due to the change of density of the sphere is given by the expression

$$
\begin{aligned}
\frac{3 g S_{i}}{(2 i+1) a}\left[\frac{1}{r^{i+1}} \int_{0}^{r}\left(i(i+1) \frac{v^{\prime}}{r^{\prime}}-\frac{1}{r^{\prime 2}} \frac{d\left({r^{\prime}}^{2} u^{\prime}\right)}{d r^{\prime}}\right) r^{i+2} d r^{\prime}\right. \\
\left.+r^{i} \int_{r}^{a}\left(i(i+1) \frac{v^{\prime}}{r^{\prime}}-\frac{1}{r^{\prime 2}} \frac{d\left({r^{\prime 2}}^{\prime} u^{\prime}\right)}{d r^{\prime}}\right) \frac{d r^{\prime}}{r^{\prime i-1}}\right] .
\end{aligned}
$$

To this must be added the potential due to the surface inequality of density $\rho$ and thickness $u_{1} S_{i}$ :

$$
\frac{3 g}{2 i+1} u_{1} \frac{r^{i}}{a^{i}} S_{i}
$$

Adding (143) and (144), and reducing by integrating by parts the last term in each definite integral, we see that the total potential may be expressed in the form

$$
\begin{aligned}
V_{i}=\frac{3 g S_{i}}{(2 i+1) a}\left[\frac { i } { r ^ { i + 1 } } \int _ { 0 } ^ { r } \left((i+1) \frac{v^{\prime}}{r^{\prime}}\right.\right. & \left.+\frac{u^{\prime}}{r^{\prime}}\right) r^{i+2} d r^{\prime} \\
& \left.+(i+1) r^{i} \int_{r}^{a}\left(i \frac{v^{\prime}}{r^{\prime}}-\frac{u^{\prime}}{r^{\prime}}\right) \frac{d r^{\prime}}{r^{i-1}}\right] .
\end{aligned}
$$

The components of bodily force due to this potential are (per unit volume)

$$
\begin{aligned}
& \rho-\frac{\partial V_{i}}{\partial r}=\frac{3 g \rho}{(2 i+1) a} {\left[-\frac{i(i+1)}{r^{i+2}} \int_{0}^{r}\left((i+1) \frac{v^{\prime}}{r^{\prime}}+\frac{u^{\prime}}{r^{\prime}}\right) r^{i+2} d r^{\prime}\right.} \\
&+\left.i(i+1) r^{i-1} \int_{r}^{a}\left(i \frac{v^{\prime}}{r^{\prime}}-\frac{u^{\prime}}{r^{\prime}}\right) \frac{d r^{\prime}}{r^{\prime i-1}}+(2 i+1) u\right] S_{i}, \\
& \frac{\rho}{r} \frac{\partial V_{i}}{\partial \theta}=\frac{3 g \rho}{(2 i+1) a}\left[\frac{i}{r^{i+2}} \int_{0}^{r}\left((i+1) \frac{v^{\prime}}{r^{\prime}}+\frac{u^{\prime}}{r^{\prime}}\right) r^{, i+2} d r^{\prime}\right. \\
&\left.+(i+1) r^{i-1} \int_{r}^{a}\left(i \frac{v^{\prime}}{r^{\prime}}-\frac{u^{\prime}}{r^{\prime}}\right) d r^{\prime}\right] \frac{\partial S_{i}}{\partial \theta},
\end{aligned}
$$




$$
\begin{aligned}
\frac{\rho}{r \sin \theta} \frac{\partial V_{i}}{\partial \phi}=\frac{3 g \rho}{(2 i+1) a} & \Gamma \frac{i}{r^{i+2}} \int_{0}^{r}\left((i+1) \frac{v^{\prime}}{r^{\prime}}+\frac{u^{\prime}}{r^{\prime}}\right) r^{i+2} d r^{\prime} \\
& \left.+(i+1) r^{i-1} \int_{r}^{a}\left(i \frac{v^{\prime}}{r^{\prime}}-\frac{u^{\prime}}{r^{\prime}}\right) d r^{\prime}\right] \frac{1}{\sin \theta} \frac{\partial S_{i}}{\partial \phi} .
\end{aligned}
$$

(4) Components of total bodily force. Combining all the bodily forces, we find

$$
\begin{aligned}
& \rho F_{r}=\rho g a\left\{-\frac{2}{3} c \frac{r^{i-1}}{a^{i}}+\frac{r}{a^{2}}\left(\frac{d u}{d r}+2 \frac{u}{r}-i(i+1) \frac{v}{r}\right)+3 \frac{u}{a^{2}}\right. \\
& +\frac{3 i(i+1)}{(2 i+1) a^{2}}\left[-\frac{1}{r^{i+2}} \int_{0}^{r}\left((i+1) \frac{v^{\prime}}{r^{\prime}}+\frac{u^{\prime}}{r^{\prime}}\right) r^{i+2} d r^{\prime}\right. \\
& \left.\left.+r^{i-1} \int_{r}^{a}\left(i \frac{v^{\prime}}{r^{\prime}}-\frac{u^{\prime}}{r^{\prime}}\right) \frac{d r^{\prime}}{r^{i-1}}\right]\right\} S_{i}, \\
& \rho F_{\theta}=\rho g a \cdot\left\{-\frac{2}{3 i} c \frac{r^{i-1}}{a^{i}}+\frac{3}{(2 i+1) a^{2}}\left[\frac{i}{r^{i+2}} \int_{0}^{r}\left((i+1) \frac{v^{\prime}}{r^{\prime}}+\frac{u^{\prime}}{r^{\prime}}\right) r^{i+2} d r^{\prime}\right.\right. \\
& \left.\left.+(i+1) r^{i-1} \int_{r}^{a}\left(i \frac{v^{\prime}}{r^{\prime}}-\frac{u^{\prime}}{r^{\prime}}\right) d r^{\prime}\right]\right\} \frac{\partial S_{i}^{\prime}}{\partial \theta},
\end{aligned}
$$

while $\rho F_{\phi}$ differs from $\rho F_{\theta}$ only in the substitution of $1 / \sin \theta \cdot \partial S_{i} / \partial \phi$ for $\partial S_{i} / \partial \theta$. These values substituted in (132), (133) and (134) give the three equations of equilibrium for a volume element. It is seen that the factors involving $\theta$ and $\phi$ cancel, also that the second and third equations are identical. Hence the problem is reduced to the solution of two simultaneous ordinary differential equations for determining $u$ and $v$ in terms of $r$.

(d) Final form of equations of interior equilibrium. We now get the equations in final form by substituting in (132) and (133) the above values of $\rho F_{\text {r }}$ and $\rho F_{\theta}^{*}$. In writing the equations we shall replace $u$ and $v$ by new functions

$$
e=\frac{u}{r}, \quad \alpha=\frac{v}{r},
$$

and introduce as independent variable $x=r / a$. The equations then take the following forms :

$$
\begin{aligned}
& (\lambda+2 \mu){ }^{2} \frac{d^{2} e}{d x^{2}}+4(\lambda+2 \mu) x \frac{d e}{d x}-i(i+1) \mu e-i(i+1)(\lambda+\mu) x \frac{d \alpha}{d x} \\
& +2 i(i+1) \mu \alpha+\rho g a\left[-\frac{2}{3} c x^{i}+\left(x \frac{d e}{d x}+6 e-i(i+1) \alpha\right) x^{2}\right. \\
& \left.+\frac{3 i(i+1)}{2 i+1}\left(-\frac{1}{x^{2+1}} \int_{0}^{x}\left[(i+1) \alpha^{\prime}+e^{\prime}\right] x^{\prime i+2} d x^{\prime}+x^{i} \int_{x}^{1}\left(i \alpha^{\prime}-e^{\prime}\right) \frac{d x^{\prime}}{x^{\prime i-1}}\right)\right]=0,
\end{aligned}
$$




$$
\begin{aligned}
& (\lambda+\mu) x \frac{d e}{d x}+(3 \lambda+5 \mu) e+\mu x^{2} \frac{d^{2} \alpha}{d x^{2}}+4 \mu x \frac{d \alpha}{d x}-\left[i(i+1) \lambda+2\left(i^{2}+i-1\right) \mu\right] x \\
& +\rho g a\left[-\frac{2 c}{3 i} x^{i}+\frac{3}{2 i+1}\left(\begin{array}{c}
i \\
x^{i+1}
\end{array} \int_{0}^{x}\left[(i+1) x^{\prime}+e^{\prime}\right] x^{\prime 2+2} d x^{\prime}\right.\right. \\
& \left.\left.+(i+1) x^{i} \int_{x}^{1}\left(i \alpha^{\prime}-e^{\prime}\right) \begin{array}{c}
d x^{\prime} \\
x^{\prime i-1}
\end{array}\right)\right]=0
\end{aligned}
$$

\section{\$3. Surface conditions.}

The stress upon the surface of the actual strained body is assumed to be 0 . This is equivalent to assuming the body to extend to or beyond the original boundary $r=a$ in every direction, and assuming the stress on this spherical surface to be a normal stress equal to the weight of the surface inequality of thickness $u_{1} S_{i}$ or $e_{1} a S_{i}, e_{1}$ being the surface value of $e$.

That this is correct to the first order of small quantities may be shown by the reasoning outlined above in the solution of the case $i=2$, which need not be here repeated. Assuming its correctness, the boundary conditions are the following:

$$
\left.\begin{array}{rl}
\widehat{r} & =-\operatorname{pgac}_{1} S_{i} \\
\widehat{r} \theta & =0 \\
r \widehat{\phi} & =0
\end{array}\right\} \text { when } x=1
$$

In these we must put

$$
\begin{aligned}
& \hat{r} r=\lambda \Delta+2 \mu \frac{\partial u_{r}}{\partial r}, \\
& \hat{r} \theta=\mu\left(\frac{\partial u_{\theta}}{\partial r}-\frac{u_{\theta}}{r}+\frac{1}{r} \frac{\partial u_{r}}{\partial \theta}\right), \\
& \hat{r} \phi=\mu\left(\frac{\partial u_{\phi}}{\partial r}-\frac{u_{\phi}}{r}+\frac{1}{r \sin \phi} \frac{\partial u_{r}}{\partial \phi}\right) .
\end{aligned}
$$

By means of (125), (126), (127) and (151) these take the forms,

$$
\begin{aligned}
& \widehat{r r}=\left[(\lambda+2 \mu) x \frac{d e}{d x}+(3 \lambda+2 \mu) e-i(i+1) \lambda \alpha\right] S_{i}, \\
& \hat{r} \theta=\mu\left(x \frac{d \alpha}{d x}+e\right) \frac{\partial S_{i}}{\partial \theta}, \\
& \widehat{r \phi}=\mu\left(x \frac{d \alpha}{d x}+e\right) \frac{1}{\sin \theta} \frac{\partial S_{i}}{\partial \phi},
\end{aligned}
$$

so that the three equations (154) reduce to the two equations, 


$$
\begin{gathered}
(\lambda+2 \mu) x \frac{d e}{d x}+(3 \lambda+2 \mu) e-i(i+1) \lambda \alpha=-\rho g a \epsilon, \\
x \frac{d \alpha}{d x}+e=0,
\end{gathered}
$$

which must be satisfied for $x=1$.

[It is easy to see what equations must replace these if different surface conditions are given; for example, if there are known surface stresses proportional to $S_{i}, \partial S_{i} / \partial \theta, 1 / \sin \theta \cdot \partial S_{i} / \partial \phi$, or given surface displacements of a similar type.]

\section{§4. Solution of equations.}

A solution of (152) and (153) satisfying (157) and (158) is obtained by assuming

$$
e=\sum A_{m} x^{m}, \quad \alpha=\sum B_{m} x^{m} .
$$

The substitution of $A_{n} x^{m}, B_{m} x^{m}$ for $e$ and $\alpha$ yields in (152) the terms

$$
\begin{aligned}
& \left\{\left[(\lambda+2 \mu)\left(m^{2}+3 m\right)-\mu i(i+1)\right] A_{m}-i(i+1)[(\lambda+\mu) m-2 \mu] B_{m}\right\} x^{m} \\
& +\rho g a\left\{\left[(m+6) A_{m}-i(i+1) B_{m}\right] x^{m+2}\right. \\
& \left.\quad+\frac{3 i(i+1)}{2 i+1}\left[-\frac{A_{m}+(i+1) B_{m}}{m+i+3} x^{m+2}+\frac{A_{m}-i B_{m}}{m-i+2} x^{m+2}-\frac{A_{m}-i B_{m}}{m-i+2} x^{i}\right]\right\},
\end{aligned}
$$

and in (153) the terms

$$
\begin{aligned}
& \left\{[(\lambda+\mu) m+(3 \lambda+5 \mu)] A_{m}+\left[\mu\left(m^{2}+3 m\right)-\left(i(i+1) \lambda+2\left(i^{2}+i-1\right) \mu\right)\right] B_{m}\right\} x^{m} \\
& +\rho g a \frac{3}{2 i+1}\left[\frac{i\left[A_{m}+(i+1) B_{m}\right]}{m+i+3} x^{m+2}\right. \\
& \left.\quad+\frac{(i+1)\left(A_{m}-i B_{m}\right)}{m-i+2} x^{m+2}-\frac{(i+1)\left(A_{m}-i B_{m}\right)}{m-i+2} x^{i}\right] .
\end{aligned}
$$

Notice first that the terms in $x^{i}$ in these two expressions are in the same ratio as the original terms of degree $i$ in (152) and (153); therefore, the terms of degree $i$ in both equations can be made to vanish by a single relation among the coefficients in the assumed series (159).

Again, for the terms of lowest degree, the coefficients of $x^{m}$ in (160) and (161) must vanish, and this is possible without the vanishing of $A_{m}$ and $B_{m}$ only if the determinant of the coefficients of $A_{m}$ and $B_{m}$ vanishes. The value of this determinant is found to be

$$
(\lambda+2 \mu) \mu(m-i)(m-i+2)(m+i+1)(m+i+3) .
$$


The four values of $m$ for which this vanishes, with the corresponding values of $B_{m} / A_{m}$, are as follows:

\begin{tabular}{|c|c|}
\hline$m$ & $\frac{B_{m}}{A_{m}}=\frac{(\lambda+2 \mu)\left(m^{2}+3 m\right)-\mu i(i+1)}{i(i+1)[(\lambda+\mu) m-2 \mu]}$ \\
\hline$i$ & $\frac{(i+3) \lambda+(i+5) \mu}{(i+1)[i \lambda+(i-2) \mu]}$ \\
$-i-2$ & $\frac{1}{i}$ \\
$-i-1$ & $-\frac{(i-2) \lambda+(i-4) \mu}{i(i+1) \lambda+i(i+3) \mu}$ \\
& $-\frac{1}{i+1}$ \\
\hline
\end{tabular}

Negative powers of $x$ being excluded by physical considerations, the series may start with terms of degree $i-2$, provided $i$ is not less than 2 . If $i$ is positive and less than 2 , the lowest degree available would be $i$.

We here assume $i$ equal to or greater than 2, and let the series (159) take the forms

$$
\begin{aligned}
& e=A_{i-2} x^{i-2}+A_{i} x^{i}+A_{i+2} x^{i+2}+\cdots, \\
& \alpha=\frac{1}{i} A_{i-2} x^{i-2}+B_{i} x^{i}+B_{i+2} x^{i+2}+\cdots
\end{aligned}
$$

From (160) and (161) it is seen that (excluding the terms of degree $i$ ) the coefficients of $x^{m+2}$ resulting from the substitution of (163) in (152) and (153) will vanish if the following equations are satisfied :

$$
[(\lambda+2 \mu)(m+2)(m+5)-\mu i(i+1)] 1_{m+2}-i(i+1)[(\lambda+\mu)(m+2)-2 \mu] B_{m+2}
$$

$$
\begin{aligned}
+ & \rho g a \frac{m^{2}+8 m-(i-3)(i+4)}{(m+i+3)(m-i+2)}\left[(m+3) A_{m}-i(i+1) B_{m}\right]=0, \\
{[(\lambda+\mu)(m+2)+3 \lambda+5 \mu] A_{m+2} } & \\
+ & \left\{\mu(m+2)(m+5)-\left[i(i+1) \lambda+2\left(i^{2}+i-1\right) \mu\right]\right\} B_{m+2} \\
& +\rho g(u)(m+i+3)(m-i+2)
\end{aligned}
$$

By means of these equations the coefficients $A_{i+2}, A_{i+4}, \ldots, B_{i+2}, B_{i+4}, \ldots$ may be expressed in terms of $\Lambda_{i}, B_{i}$, leaving thus far arbitrary the three constants $\alpha_{i-2}, A_{i}, B_{i}$.

It remains only to equate to zero the coefficients of $x^{i}$, which as already 
remarked requires but a single equation. The two differential equations will thus be satisfied by values of $e$ and $\alpha$ of the assumed form, containing two arbitrary constants, provided the series thus determined prove to be convergent. The two arbitrary constants suffice for the satisfaction of the surface conditions.

The solution of (164) and (165) gives

$$
\begin{aligned}
A_{m+2}=- & \frac{\rho g a}{\lambda+2 \mu} \\
& \times \frac{[(m+4)(m+6) \mu-i(i+1)(\lambda+2 \mu)]\left[(m+3) A_{m}-i(i+1) B_{m}\right]}{(m-i+2)(m+i+5)(m-i+4)(m+i+3) \mu},
\end{aligned}
$$

$$
\begin{aligned}
& B_{m+2}=\frac{\rho g a[m(\lambda+\mu)+5 \lambda+4 \mu]\left[(m+3) A_{m}-i(i+1) B_{m}\right]}{(\lambda+2 \mu)(m-i+2)(m+i+5)(m-i+4)(m+i+3) \mu}, \\
& \frac{(m+5) A_{m+2}-i(i+1) B_{m+2}}{(m+3) A_{m}-i(i+1) B_{m}}=-\frac{m+6}{(m-i+2)(m+i+3)} \cdot \frac{\rho g a}{\lambda+2 \mu}
\end{aligned}
$$

By means of these equations the values of all coefficients after the first two in each of the assumed series (163) may be expressed in terms of $A_{i}$ and $B_{i}$. These values may be expressed as follows, $n$ taking values $0,1,2, \ldots$ :

$$
\begin{aligned}
A_{i+2 n+2}=(-b)^{n+1}\left[i(i+1) \frac{\lambda}{\mu}+2 i(i+1)-(i+2 n+4)(i+2 n+6)\right] & (i+4)(i+6) \cdots(i+2 n+4)\left[i(i+1) B_{i}-(i+3) A_{i}\right] \\
& \times(i+4)[2 \cdot 4 \cdots(2 n+4)][(2 i+3)(2 i+5) \cdots(2 i+2 n+5)] \\
B_{i+2 n+2}=(-b)^{n+1}\left[(i+2 n+5) \frac{\lambda}{\mu}+i+2 n+4\right] & {\left[(i+4)(i+6) \cdots(i+2 n+4)\left[i(i+1) B_{i}-(i+3) A_{i}\right]\right.} \\
& \times \frac{(i+4)[2 \cdot 4 \cdots(2 n+4)][(2 i+3)(2 i+5) \cdots(2 i+2 n+5)]}{(i+2 n+1} \cdot
\end{aligned}
$$

The coefficient of $x^{i}$ may be found by substituting in (160) or (161) successively $m=i-2, i, i+2, \ldots$, remembering that $i B_{i-2}=A_{i-2}$, and that, as already pointed out, a single equation causes the vanishing of the coefficients of $x^{i}$ in both (15\%) and (153). This equation may be written in the following form, after dividing throngh by $\lambda+2 \mu$ and writing $b$ for $g \rho a /(\lambda+2 \mu)$ :

$$
\begin{aligned}
& i(i+1)\left[\left(\frac{\lambda+\mu}{\lambda+2 \mu}+\frac{2}{i+1}-\frac{3 b}{2(2 i+1)}\right) A_{i}-\left(\frac{i \lambda+(i-2) \mu}{\lambda+2 \mu}-\frac{3 i b}{2(2 i+1)}\right) B_{i}\right] \\
& +\frac{3 i b}{2 i+1} A_{i-2}-\frac{3 i(i+1) b}{2 i+1} \sum_{0}^{\infty} \frac{A_{i+2+2 n}-i B_{i+2+2 n}}{2 n+4}-\frac{2}{3} b c=0 .
\end{aligned}
$$

Aiso, from (169) and (170), 


$$
\begin{aligned}
& \frac{A_{i+2+2 n}-i B_{i+2+2 n}}{2 n+4}=(-b)^{n+1}\left[i_{-\mu}^{\lambda}+3 i+2(n+3)\right] \\
& \times \frac{[(i+4)(i+6) \cdots(i+2 n+4)]\left[(i+3) A_{i}-i(i+1) B_{i}\right]}{(i+4)[2 \cdot 4 \cdots(2 n+4)][(2 i+3)(2 i+5) \cdots(2 i+2 n+5)]},
\end{aligned}
$$

by means of which (171) is reduced to a relation among the three constants $A_{i-2}, A_{i}, B_{i}$.

The differential equations (152) and (153) are thus satisfied by (163), provided the coefficients satisfy (169), (170), (171) and (172); and two of the coefficients still remain arbitrary. By properly assigning these, the surface conditions (157) and (158) may be satisfied. The solution may be expressed in fairly compact form by means of the following functions :

$$
\begin{aligned}
L(z)= & \sum_{0}^{\infty}(-1)^{n}\left[i(i+1) \frac{\lambda}{\mu}+2 i(i+1)-(i+2 n+4)(i+2 n+6)\right] \\
& \times \frac{(i+4)(i+6) \cdots(i+2 n+4)}{(i+4)[2 \cdot 4 \cdots(2 n+4)][(2 i+3)(2 i+5) \cdots(2 i+2 n+5)]} z^{n}, \\
M(z)= & \sum_{0}^{\infty}(-1)^{n}\left[(i+2 n+5) \frac{\lambda}{\mu}+i+2 n+4\right] \\
& \times \frac{(i+4)(i+6) \cdots(i+2 n+4)^{\prime}}{(i+4)[2 \cdot 4 \cdots(2 n+4)][(2 i+3)(2 i+5) \cdots(2 i+2 n+5)]} z^{n}, \\
N(z)= & \sum_{0}^{\infty}(-1)^{n}\left[i{ }^{\lambda}+3 i+2(n+3)\right] \\
& \times \frac{(i+4)(i+6) \cdots(i+2 n+4)}{(i+4)[2 \cdot 4 \cdots(2 n+4)][(2 i+3)(2 i+5) \cdots(2 i+2 n+5)]} z^{n}, \\
L^{\prime}(z)= & \sum_{0}^{\infty}(-1)^{n}\left[i(i+1) \frac{\lambda}{\mu}+2 i(i+1)-(i+2 n+4)(i+2 n+6)\right] \\
& \times \frac{(i+2 n+2)[(i+4)(i+6) \cdots(i+2 n+4)]}{(i+4)[2 \cdot 4 \cdots(2 n+4)][(2 i+3)(2 i+5) \cdots(2 i+2 n+5)]} z^{n}, \\
M^{\prime}(z)= & \sum_{0}^{\infty}(-1)^{n}\left[(i+2 n+5) \frac{\lambda}{\mu}+i+2 n+4\right] \\
& \times \frac{(i+2 n+2)[(i+4)(i+6) \cdots(i+2 n+4)]}{(i+4)[2 \cdot 4 \cdots(2 n+4)][(2 i+3)(2 i+5) \cdots(2 i+2 n+5)]} z^{n} .
\end{aligned}
$$

The values of $e$ and $\alpha$, and the three equations for determining $A_{i-2}, A_{i}$ and $B_{i}$, may then be written as follows, the third equation being equivalent to (171), the fourth to (157), and the fifth to (158): 
(A) $e=A_{i-2} x^{i-2}+A_{i} x^{i}+\left[(i+3) A_{i}-i(i+1) B_{i}\right] b x^{i+2} L\left(b x^{2}\right)$,

(B) $\quad \alpha=\frac{1}{i} A_{1-2} x^{i-2}+B_{i} x^{i}+\left[(i+3) A_{i}-i(i+1) B_{i}\right] b x^{i+2} M\left(b x^{2}\right)$,

$(C)$

$$
\frac{3 i b}{2 i+1}\left(A_{i-2}+A_{i}\right)+\frac{(4 i+6) \mu}{\lambda+2 \mu} A_{i}
$$

$$
+\left[(i+3) A_{i}-i(i+1) B_{i}\right]\left(\frac{i \lambda+(i-2) \mu}{\lambda+2 \mu}-\frac{3 i b}{2(2 i+1)}+\frac{3 i(i+1)}{2 i+1} b^{2} N(b)\right)-\frac{2}{3} b c=0,
$$$$
\left[b\left(\begin{array}{l}
\lambda \\
\mu
\end{array}+2\right)+2 i-2\right]\left(A_{i-2}+A_{i}\right)+4 A_{i}+\left[(i+3) A_{i}-i(i+1) B_{i}\right]
$$

(D)

$$
\begin{aligned}
\times\left[\frac{\lambda}{\mu}+\left(\frac{\lambda}{\mu}+2\right) b L^{\prime}(b)+\left(3 \frac{\lambda}{\mu}+2\right) b L(b)\right. \\
\left.+\left(\frac{\lambda}{\mu}+2\right) b^{2} L(b)-i(i+1) \frac{\lambda}{\mu} b M(b)\right]=0,
\end{aligned}
$$

$$
\begin{aligned}
\left(i^{2}-1\right)\left(A_{i-2}+A_{i}\right) & +(2 i+1) A_{i}+\frac{i}{2}\left[(i+3) A_{i}-i(i+1) B_{i}\right] \\
& \times\left[-1+(i+1) b L(b)+(i+1) b M^{\prime}(b)\right]=0 .
\end{aligned}
$$

These correspond to equations $(A),(B),(C),(D),(E)$ obtained in Part I of this paper, and reduce to them when $i=2$. It must be noted, however, that the notation is slightly different, $e, \alpha$ and the functions (173) differing by constant factors from the corresponding functions in Part $I$. 